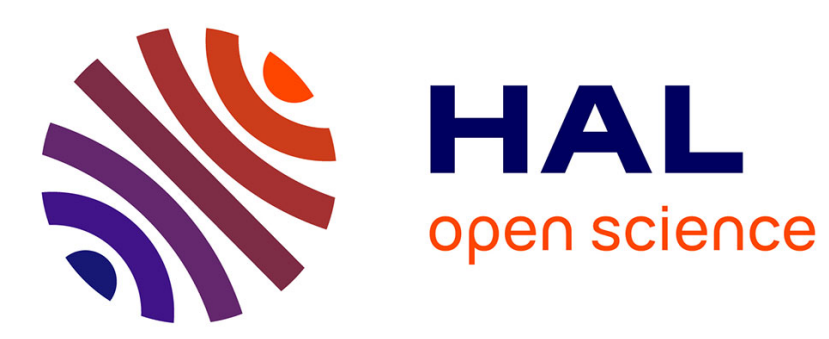

\title{
PROLETARIAT COMBATIF, OUVRIERS ATONES: LE PARADOXE SINGAPOURIEN (1936-1995)
}

\author{
Margolin Jean-Louis
}

\section{To cite this version:}

Margolin Jean-Louis. PROLETARIAT COMBATIF, OUVRIERS ATONES: LE PARADOXE SINGAPOURIEN (1936-1995). Le Mouvement social, 1995. hal-01935642

\section{HAL Id: hal-01935642 \\ https://hal.science/hal-01935642}

Submitted on 26 Nov 2018

HAL is a multi-disciplinary open access archive for the deposit and dissemination of scientific research documents, whether they are published or not. The documents may come from teaching and research institutions in France or abroad, or from public or private research centers.
L'archive ouverte pluridisciplinaire HAL, est destinée au dépôt et à la diffusion de documents scientifiques de niveau recherche, publiés ou non, émanant des établissements d'enseignement et de recherche français ou étrangers, des laboratoires publics ou privés. 
(Le mouvement social, $\mathrm{n}^{\circ}$ "Les dynamiques ouvrières en Asie orientale", 173, octobredécembre 1995, pp. 113-148)

\section{PROLETARIAT COMBATIF, OUVRIERS ATONES: LE PARADOXE SINGAPOURIEN (1936-1995)}

Le Singapour high tech et prospère des années quatre-vingt dix offre avec la colonie britannique des années cinquante un contraste saisissant. Le passage d'un mouvement ouvrier actif aux travailleurs passifs et muets d'aujourd'hui ne l'est pas moins. Entre 1959 et 1963, le "Cuba asiatique" que d'aucuns redoutaient connut pour la dernière fois des luttes sociales d'ampleur considérable. On en était en fait, en un gros quart de siècle, à la quatrième vague de grèves et de (re)construction d'un mouvement syndical puissant -et communisant. La première (1936-40), écho de l'ébullition sur les plantations et dans les mines de Malaisie, constitua le point de départ du syndicalisme militant, indissolublement lié dès lors, pour le meilleur et pour le pire, aux destinées du parti communiste local ${ }^{12}$; dès 1940, la General Labour Union (GLU) clandestine regroupait, avec vingt mille adhérents, le cinquième des salariés. La seconde vague (1945-48), centrée elle sur le grand port, fut à la fois plus politically centred, socialement plus ambigüe, mais aussi encore plus ample:150 000 grévistes le 30 janvier 1946, soit plus que le total des salariés; adhésion dans les deux ans qui suivirent de plus de 50\% de ceux-ci à la Pan Malayan General Labour Union -PMGLU- communisante; d'avril 1946 à mars 1947, on décompta 1,2 million de journées de grève, soit dix par salarié. La troisième vague (1954-56) représenta la relance du mouvement social et syndical, après les terribles coups portés à tout ce qui ressemblait de loin à un communiste lors des premières années de 1'Emergency (1948-1960); elle accompagna aussi l'entrée de Singapour en self government, lors des élections législatives de 1955. Elle vit la proportion des syndiqués atteindre $35 \%$ à $40 \%$ de la main d'oeuvre - comme dans la période de luttes suivante. Celle-ci, entre 1961 et 1963 fut le champ du cygne tout à la fois du prolétariat "ancien", du syndicalisme indépendant du pouvoir et de la prépondérance politique du communisme (clandestin) à l'échelle de la société entière; elle ne put prévenir ni, à court terme, l'unification avec la Malaisie, ni, à plus long terme, la consolidation d'un pouvoir conservateur et fort qui bientôt entreprit de démanteler toute forme d'expression hors-normes.

Or, depuis 1978, la grève paraît presque rayée de la carte des possibles; quelques centaines de journées de travail seulement ont été "perdues". Bien plus, la confédération syndicale National Trade Union Congress (NTUC), pourtant située clairement dans les allées du pouvoir, voit ses adhérents osciller entre $15 \%$ et $20 \%$ du public visé, soit moitié

1

${ }^{2} \mathrm{Ne}$ reconnaissant pas la légitimité du partage colonial entre colonie singapourienne et protectorats malais, il opère sous ce nom sur les deux territoires 
moins que dans les syndicats du début des années soixante. L'atmosphère est à une pesante contention, le discours à un "tout économique" faisant fi des préoccupations sociales. Très peu de syndicalistes d'opposition ont cependant effectué de longs séjours en prison, et ce pouvoir oppressif n'est point meurtrier. Tout se passe comme s'il s'était produit une profonde mutation dans les consciences, comme si, par une opération collective d'amnésie, tout un passé de combativité s'était trouvé aboli.

L'impression d'étrangeté est encore accentuée par l'évolution du secteur industriel. Jusqu'aux alentours de l'indépendance (août 1965), il était globalement faible, en comparaison non seulement de celui des grands centres urbains des pays développés, mais même à celui d'un Shanghaï, d'un Mexico ou d'un Calcutta. Les manufactures ne fournissaient du travail en 1947 qu'à $17 \%$ des actifs $\left(\begin{array}{lll}59 & 000\end{array}\right.$ personnes), en 1957 qu'à $77000(16,3 \%)$ - ce qui est très peu compte tenu de la grande faiblesse (déjà alors moins de $10 \%$ des actifs) du secteur primaire, dans une île où l'espace est la denrée la plus précieuse; avec les travailleurs du bâtiment $(24500)$, on frôle péniblement les 100000 (21,5\% des actifs). Encore la plupart des ouvriers devaient-ils travailler dans de minuscules entreprises, souvent proches de l'artisanat: le premier recensement industriel, en 1959, ne décèle dans les usines de dix salariés au moins que 27400 ouvriers soit moins de $40 \%$ des effectifs mentionnés deux ans plus tôt; bien moins aussi que les quelque 36000 employés civils des bases militaires britanniques de 1960. On peut aller plus loin: le recensement de 1957 relève $31,3 \%$ de Craftsmen, production process workers, and labourers et 8,3\% de Transport and communication workers ${ }^{3}$ (c'est à dire 39,6\% -186 000 personnes- de travailleurs manuels salariés non ou moyennement qualifiés) dans la population active ${ }^{4}$. A peine $40 \%$ de ceux-ci travaillent donc dans l'industrie: les dockers, les manutentionnaires, les coolies, les ouvriers des transports et du bâtiment, etc, sont globalement aussi nombreux que les ouvriers d'usine.

La situation a été complètement bouleversée par une industrialisation aussi ample que rapide, amorcée vers 1967. Dès 1980 (année à partir de laquelle le secteur secondaire va "plafonner" relativement parlant, dans une économie qui demeure très expansive) on compte 313000 salariés dans l'industrie (29,2\% des actifs), 58000 dans le bâtiment (5,4\%), soit au total 371000 (34,6\%). Sur les 415000 travailleurs manuels non ou moyennement qualifiés (38,8\% des actifs), 255000 (23,9\% des actifs) travaillent dans l'industrie - soit $61 \%$ de l'effectif. De plus les moyennes et grandes entreprises (celles qui disposent de dix salariés au moins) emploient désormais plus des neuf

${ }^{3}$ Les deux catégories sont désormais réunies sous l'appellation Production craftsmen, plant and machine operators, cleaners and labourers (cf Ministry of Trade and Industry, Economic survey of Singapore 1994, Singapour, SNP Publishers, 1995) 4You Poh Seng et al., "Population growth and population characteristics", in You Poh Seng \& Lim Chong Yah, The Singapore economy, Singapour, Eastern Universities Press, 1971, pp. 68-69; Chia Siow Yue, "Growth and pattern of industrialization", ibid., p. 222 
dixièmes des salariés de l'industrie (289 000 sur 313 000); leur main d'oeuvre ouvrière a ainsi été multipliée par huit au moins en un petit quart de siècle. L'industrialisation est la grande responsable (avec la transition démographique, qui accroît relativement le nombre d'adultes jeunes) de la mise au travail massive de la population, tout particulièrement féminine: $28,4 \%$ d'actifs en $1957,44,7 \%$ en $1980^{5}$.

L'évolution est donc à première vue très paradoxale: dans un premier temps, une combativité, un poids politique très forts alors que, sociologiquement, la classe ouvrière "classique" est pratiquement introuvable; un effacement à peu près total, alors même que les ouvriers d'industrie deviennent une masse déterminante, comparable numériquement aux situations, sinon de l'Europe occidentale, du moins de celles du Japon ou de l'Amérique du Nord. Bref, pour reprendre la vieille terminologie d'origine hégelienne, une classe "pour soi" qui paraît cesser d'exister quand surgit la classe "en soi"...

De cela on peut effectuer deux lectures. La première, historique (et même un peu historiciste), est la seule à avoir été développée, essentiellement par la (courte) bibliographie critique à l'égard de l'actuel pouvoir singapourien: elle dénonce l'entreprise systématique d'extirpation de toute conscience de classe, de toute capacité d'action autonome; et il est vrai que les arguments ne manquent pas. La seconde, sociologique, est à peine esquissée, indirectement, par de très rares auteurs; il est vrai que, plus généralement, la structure sociale d'un pays pourtant peu avare en statistiques et moyens d'investigations est incroyablement peu étudiée: les quelques études pionnières sur la pauvreté, le logement, les clivages sociaux des années cinquante et du début des années soixante ${ }^{6}$ n'ont pratiquement pas eu à ce jour d'héritières - marque de ce qu'on touche sans doute là à certains des tabous majeurs du "système Singapour", en particulier à son discours d'homogénéité absolue, où les seules déviances intellectuellement admissibles seraient individuelles ${ }^{7}$.

Cette piste doit cependant être suivie, malgré la pauvreté des données disponibles: la majeure partie des ouvriers n'ont été, ne sont ni ces militants, ni ces syndicalistes, ni ces grévistes, ni, en sens inverse, ces suivistes zélés des mots d'ordre patronaux et gouvernementaux, qui ont concentré toute l'attention des policiers, des dirigeants d'entreprise, des cadres politiques (y compris ceux de la gauche radicale) ou syndicaux - et, à leur suite, des auteurs, souvent personnellement liés à eux. Pour percevoir dans leur évolution ces masses obscures, on dispose quand même de l'analogie, mieux étudiée, des ouvriers des

${ }^{5}$ Economic Survey, années1980 et 1994

${ }^{6}$ Barrington Kaye, Upper Nankin Street: A sociological study of Chinese households living in a densely populated area, Singapour, University of Malaya Press, 1960; Goh Keng Swee, Urban Incomes and Housing: A report on the social survey of Singapore, 1953-54, Singapour, Government Printing Office, 1956

${ }^{7}$ C'est peut-être là un marqueur fondamental de ce qu'on pourrait dénommer, malgré toutes les imperfections et ambigüités du terme, phénomène totalitaire. 
"vieux" pays industriels, et de France en particulier: même si le phénomène y fut moins net (mais la révolution industrielle y fut aussi considérablement plus lente), on y constate aussi, au XIXème siècle, cette combinaison d'un grand radicalisme de la pensée et de l'action, qui ne s'appuyait que marginalement sur des ouvriers "de masse", et de la croissance d'une population manufacturière longtemps politiquement et syndicalement invisible. On tend désormais, d'une part à dissocier fortement, au moins jusqu'aux alentours de 1914, histoire des ouvriers et histoire du socialisme (y compris dans ses variantes anarchiste et syndicaliste); d'autre part à remettre en cause l'idée même d'une vocation contestatrice des travailleurs industriels, en particulier à la lumière de leur quasi-absence de la scène politique et de la faiblesse des luttes sociales depuis le début de l'actuelle dépression économique, en France comme dans la plupart des vieux pays industriels. Révolution copernicienne de la sociologie: elle insiste aujourd'hui sur l'extrême atomisation, la diversité, l'instabilité, la fragilité des structures (mentales autant que matérielles) de ce qu'on n'ose plus appeler classe ouvrière. La question centrale sera au fond ici de distinguer prolétaires (concept et réalité d'abord d'ordre politique) et ouvriers (entité avant tout socio-économique), en se demandant si la transformation des premiers dans les seconds ne rend pas aussi bien compte que la main de fer de l'Etat d'une évolution dérangeante.

\section{I - Première configuration: un prolétariat politisé (1936-1965)}

Les trente années qui encadrent le milieu du siècle sont pour Singapour la "zone des tempêtes". Jusque là, une prospérité quasiment continue, à peine inquiétée par de courtes crises comme la dépression du caoutchouc en 1920-21, voyait les matières premières d'exportationreines se succéder les unes aux autres, et les flots d'immigrants, indiens et chinois surtout, aller et venir librement entre leur terre d'origine, le grand port et les plantations et mines d'Asie du Sud-Est, au seul gré de l'offre de travail, de la conjoncture des pays de départ et de leur trajectoire personnelle, où le retour au village était souvent inscrit: 242000 Chinois débarquèrent à Singapour au cours de la seule année 1930, alors que, pour la décennie 1921-1931, le surplus migratoire total n'est que de $124000^{9}$. En 1931, sur 567000 sino-singapouriens, 358000 étaient nés en Chine $^{10}$, et y avaient généralement vécu jusqu'à l'âge adulte.

C'est alors que surgit localement la crise mondiale, avec une extrême violence (le cours des matières premières locales baisse en moyenne de 60\%). Les dizaines de milliers de licenciements expliquent cette mesure sans précédent: le blocage de l'immigration, pour les

${ }^{8}$ Gérard Noiriel, Les ouvriers dans la société française - XIXè-XXè siècle, Paris, PointsSeuil, 1986; Christophe Charle, Histoire sociale de la France au XIXè siècle, Paris, Points-Seuil, 1991

${ }^{9}$ David C.E. Chew, Amina H. Degani, "Population and Manpower", in Ooi Jin-bee et Chiang Hai Ding (ed.), Modern Singapore, University of Singapore, 1969, p. 86 ${ }^{10}$ Jean-Louis Margolin, Singapour 1959-1987: genèse d'un nouveau pays industriel, Paris, L'Harmattan, 1989, p.18 
Chinois masculins dès 1931, pour les Chinoises en 1937. Les troubles politico-militaires qui relayent immédiatement, et pour longtemps, les difficultés économiques achèvent de pérenniser une mesure de circonstance. Ses conséquences sont fondamentales: les immigrants sont "cloués" sur place, y font souche (dès 1954, les Chinois local-born sont majoritaires), et se montrent bien plus qu'auparavant politiquement ou syndicalement mobilisables. En effet, outre que leur réussite ou leur échec se joue désormais sur place, les tempséconomiques autant que politiques- sont particulièrement durs. De plus ils ne sont pas, dans leur majorité, depuis suffisamment longtemps dans le Nanyang ${ }^{11}$ pour se désintéresser des grandioses évènements, tour à tour dramatiques et exaltants, qui touchent leur patrie d'origine, qu'elle soit l'Inde, l'Indonésie ou la Chine. C'est sur la base de leurs frustrations, de leurs angoisses, de leurs espoirs, sur la base aussi de la grande précarité de leur condition, plus que sur une communauté de situation sociale, que les plus actifs de ces prolétaires vont se lancer, à quatre reprises en trente ans, à l'assaut du système dont ils ressentent l'oppression.

\section{Un prolétariat plus qu'une classe ouvrière}

Jusqu'avant dans les années soixante, la misère est sans doute à Singapour la chose la mieux partagée. Aux normes asiatiques du temps, elle n'est pas extrême (la colonie dispose même au début des années cinquante du revenu par tête le plus élevé d'Asie, devant le Japon lui-même), mais le quart des Singapouriens n'atteint pas en 1953-54 un minimum vital pourtant calculé au plus juste. Certaines "niches" sont particulièrement défavorisées: les familles nombreuses (40\% au dessous du seuil de pauvreté), les veuves avec enfants (51\%), les couples chargés de parents (35\%), les plus de 60 ans (36\%), les femmes (28\% chez les 18-59 ans, pour 19\% des hommes du même âge), la quasi-totalité des $3 \%$ à $5 \%$ de chômeurs, dont $57 \%$ n'ont pas la moitié du minimum vital ${ }^{12}$.

Deux points surtout aident à comprendre la conscience "prolétarienne" aïgue qui se développe. D'une part, sur un terrain aussi sensible en milieu urbain que le logement, la détérioration est très nette, rapide, et la situation dramatique: la surface bâtie totale par habitant baisse de $29 \%$ entre 1890 et $1950^{13}$; pour les ménages de huit personnes, la concentration par pièce passe de 4,1 en 1947 à 4,8 en 1954; $71 \%$ des ménages de cinq à huit personnes, $27 \%$ de ceux de onze personnes vivent dans une seule pièce; $24 \%$ des foyers couchent sur un banc, sous un escalier, dans un bout de couloir, sur un pas de porte ${ }^{14}$. A Upper Nankin street (au coeur de Chinatown), on compte jusqu'à 83

\footnotetext{
11"Mer du sud" en chinois, c'est à dire "notre" mer de Chine du Sud; par extension l'ensemble des terres qui en sont proches, c'est-à-dire l'Asie du Sud-Est ${ }^{12}$ Goh Keng Swee, Urban incomes and housing: a report on the social survey of Singapore 1953-1954, Singapour, Government Printing Office, 1956, p. 113-129 ${ }^{13}$ Rory Fonseca, "Planning and land use", in Riaz Hassan (ed.), Singapore: Society in Transition, Kuala Lumpur, Oxford University Press, 1976, p. 223

${ }^{14}$ Goh, op. cit., pp. 68 et 73
} 
locataires dans les étroites shop-houses de deux étages; chaque "équivalent adulte" (l'enfant de moins de dix ans compte pour moitié) dispose d'un peu moins de $4 \mathrm{~m} 2$; on compte en moyenne quinze adultes par robinet, vingt par $\mathrm{WC}^{15}$.

D'autre part, si $15 \%$ des salariés gagnent moins que le minimum vital, c'est le cas de $26 \%$ des travailleurs indépendants: les marchands des quatre saisons (ou hawkers), les petits artisans, les conducteurs de cyclopousses ${ }^{16}$. Le revenu mensuel moyen est de 140 dollars locaux; on trouve nettement au-dessus l'ensemble des cols blancs (dactylos: 213\$; infirmières: 206\$), mais en dessous tant les hawkers (125\$) que les ouvriers non qualifiés (110\$) ou les manoeuvres (81\$); les autres indépendants gagnent entre 140 et $150 \$ 17$. Comme dans beaucoup de pays du tiersmonde ${ }^{18}$, comme aussi dans le Paris de la Révolution française, ou même dans la France de la seconde moitié du dixneuvième siècle ${ }^{19}$, la distinction est nette entre ceux qui travaillent de leurs mains et les autres, mais très brouillée (dans la réalité, et plus encore dans les consciences) entre salariés, indépendants, tout petits patrons et agriculteurs, alors encore relativement nombreux (environ $8,5 \%$ des actifs en 1947, pour la plupart des Chinois). Ils forment, ensemble, la vaste masse aux contours vagues d'un peuple -comme on disait vers 1789 - que nous préférerons dénommer prolétariat, à la fois parce qu'ils vont s'unir, l'espace d'une génération, autour du message "prolétarien" moderne propagé par le Parti Communiste de Malaisie (PCM); mais aussi parce que beaucoup d'entre eux, bien plus que les Sans-Culottes décrits par Albert Soboul, n'ont littéralement rien à eux: ni logement digne de ce nom, on l'a vu, ni sépulture familiale proche, ni surtout famille pour bon nombre des plus âgés $(1,7$ homme pour une femme en 1921; 1,2 en 1947), hommes le plus souvent, mais aussi servantes (amah) ou terrassières de la "caste" célibataire Shaam Shui. Il faudrait aussi évoquer les chômeurs, évidemment non aidés, qui passent de 5-8\% en 195420 à $15 \%$ environ en 1962-63.

Cette absence de centralité ouvrière est implicitement admise par les communistes eux-mêmes. Ainsi, parmi les adhérents les plus importants de la GLU de 1945, on trouve la Hawkers Union, la Goldsmiths Guild, la Chinese and Western Medicinal Trade Workers Mutual Aid Society, la Barbers Union, la Farmers Union, et encore des associations de pêcheurs, d'agriculteurs à temps partiel, d'employés de commerce, de cyclopousses, etc. Les syndicats les plus nombreux sont ceux des dockers, des conducteurs de bus, des employés de la base

\footnotetext{
${ }^{15}$ Barrington Kaye, Upper Nankin street: A sociological study of Chinese households living in a densely populated area, Singapour, University of Malaya Press, 1960, ch. 5b

${ }^{16}$ Goh, op. cité, pp. 136-143

${ }^{17}$ Ibid., pp. 98 et 194

${ }^{18}$ Sur les raisons de cette graphie, cf EspacesTemps, n45-46 (Sortir du

tiersmonde: système-monde, nouvelles figures), 1991, éditorial

${ }^{19} \mathrm{Cf}$ Christophe Charle, op. cité

${ }^{20}$ Les statistiques sont là particulièrement incertaines
} 
navale. Dans ce surgeon final du syndicalisme radical qu'est entre 1961 et 1963 la Singapore Association of Trade Unions (SATU), les hawkers sont à nouveau très présents, mais on note un surgissement particulièrement considérable d'associations culturelles ou athlétiques, ainsi que de ces locataires particuliers que sont les Squatters ${ }^{21}$ ou les Attap $^{22}$ House Dwellers. Les regroupements ne se font donc pas du tout systématiquement sur la base du métier, mais tout autant sur celle du logement ou des activités choisies. De plus la diversité des appellations associatives (syndicat, mais aussi guilde, société de secours mutuel...) évoque une prise en charge de la très vivante sociabilité chinoise traditionnelle, celle des bang (23 $^{3}$, ces "familles de substitution" pour tous les isolés, les orphelins du culte des ancêtres, les nostalgiques du district ou du clan d'origine. En ce sens, et même s'il convient de ne pas pousser l'analogie trop loin, les confédérations syndicales "rouges" sont les héritières des hui, les sociétés secrètes interdites depuis les années 1880, et désormais enkystées dans le banditisme.

Quant aux principaux clivages, ils se situent entre un secteur public où, à l'exception du port, les syndicats et les associations amicales $^{24}$ modérés dominent largement; un secteur privé européen, alors essentiellement commercial, où dominent moyennes et grandes entreprises; et un secteur privé asiatique, presque entièrement chinois, et composé avant tout de petites sociétés. Les différences d'activité sont moins pertinentes que les clivages culturels (english-educated dans les deux premiers secteurs, chinese-educated dans le dernier) et, dans une large mesure, les logiques ethniques (les Indiens sont très nombreux dans le secteur public, les Eurasiens dans le privé européen; la plupart des entreprises chinoises n'emploient que des Chinois).

L'industrie ne constitue dans cette configuration complexe qu'un ensemble modeste: PME presque sans exception (la plus grande usine, en 1962, est une fonderie d'étain), faible mécanisation, marché essentiellement local - tout particulièrement dans les secteurs alors pratiquement captifs, compte tenu des coûts rédhibitoires du transport rapide à longue distance, que constituent le ravitaillement quotidien et la première transformation des produits primaires exportés. On compte ainsi en 19596200 ouvriers dans l'alimentaire (y compris la boisson), 3900 dans l'imprimerie, 2800 dans le bois et l'ameublement, ainsi que dans la transformation des minerais (ce sont les quatre principales branches) mais seulement 2000 dans le textile et les chaussures, 1400 dans les machines, 1100 dans l'équipement électrique ${ }^{25}$. Ces industries de main d'oeuvre sont contrôlées par des capitaux essentiellement asiatiques: à 60\% dans les ateliers de dix ouvriers au moins.

\footnotetext{
${ }^{21}$ Tous les occupants sans titre sont dénommés ainsi, y compris beaucoup d'agriculteurs

${ }^{22}$ Toit de "chaume" local; désigne un habitat largement spontané, médiocre, précaire

${ }^{23} \mathrm{Cf}$ Cheng Lim-keak, Social change and the Chinese in Singapore, Singapore

University Press, 1985

${ }^{24}$ Les fonctionnaires n'ont pas alors le droit de se syndiquer

${ }^{25}$ Report on the Census of Industrial Production, 1959, cité in Jean-Louis Margolin, op. cité, p. 20
} 
Corporatisme et hyper-politisation: le double écueil

Le caractère irréductiblement hétérogène du prolétariat singapourien explique que son unité ne puisse se réaliser que sur des objectifs politiques très généraux, correspondant plus à une sensibilité qu'à des intérêts. Tant qu'on se situe sur le terrain de ces derniers -et donc essentiellement dans l'économico-social-, les divergences et contradictions dominent, et les luttes tendent à se parcellariser autour de revendications corporatistes. On s'en prend rarement au patronat en général, et même aux patrons en particulier. Les grèves, parfois violentes, de 1936-37, ou de l'après-1945, sont fréquemment dirigées contre les "marchandeurs" de main d'oeuvre (subcontractors dans les usines et sur les chantiers navals, kangany-s sur les plantations peuplées d'Indiens) ou contre les contremaîtres-potentats, de plus accusés au lendemain de l'Occupation de s'être comportés en "collabos" des Japonais. On conteste la discipline semi-militaire qui règne sur l'énorme base navale. On s'en prend à tel ou tel service administratif, accusé d'interventionnisme excessif, ou au contraire d'indifférence. Les revendications sont très diverses, très locales. Les autorités coloniales en jouent souvent pour se défausser de toute responsabilité sur les "employeurs chinois peu scrupuleux"26: la police intervient principalement quand se produisent des débordements dans la rue. Les patrons chinois jouent eux-mêmes sur les solidarités - et oppositionsethniques, dialectales, voire claniques. Ainsi la GLU reste longtemps confinée aux Hailam-s de lî̀le de Haïnan, très minoritaires parmi les Chinois mais très radicaux car derniers arrivants et méprisés comme "semi-barbares" par les Cantonais et Fukienois mieux installés: en 1933, elle compte 5600 Hailam-s sur 7000 membres. A contrario on dénote fin 1937 l'adhésion en bloc à la GLU d'une cinquantaine de couturières Foochow. En juin 1937, le PCM doit passer par une première version anglaise pour traduire sa propagande du chinois au tamoul. Et, à Ho Hong Soap Factory, les communistes tamouls refusent de faire grève avec les communistes chinois.

La logique de la lutte tend cependant à fortifier l'unité entre syndicats et entre catégories de travailleurs, à éliminer les associations de jaunes (comme lors de la grande grève de la réparation navale, en janvier 1940), à susciter des revendications unifiantes: ainsi la GLU tente-t-elle en octobre 1936 d'organiser de façon permanente les ouvriers du bâtiment à l'échelle de la Malaisie entière, à l'aide d'une série d'accords avec les associations locales. Mais les militants (il est vrai sollicités par la conjoncture) semblent ne pouvoir résister à la solution de facilité que constitue la politisation à outrance. Il est relativement aisé au moment des agressions japonaises contre la Chine de susciter de vastes mouvements de solidarité, à façade légale (ils sont nominalement dirigés par des hommes d'affaires "patriotes", dignitaires

\footnotetext{
${ }^{26}$ Straits Settlements Police Intelligence Journal (SSPIJ), publication mensuelle classifiée de la Special Branch (police politique britannique) de Singapour; octobre 1936. Sauf mention contraire, les informations concernant l'avant-guerre proviennent de cette source riche et très informée.
} 
de la Chambre de Commerce chinoise, tels que le légendaire Tan Kah $\mathrm{Kee}^{27}$, qui finira par rallier le PC chinois): à travers le National Salvation Movement, version locale du Front Uni Anti-Japonais qui suit l'incident de Xian (1936), la GLU peut atteindre en 1940 les 20000 adhérents (certaines sources lui en attribuent même 40 000), organiser le 1er Mai 1940 , après de multiples échecs, la première grève générale quasi-totale de 1'histoire de la colonie, et réunir 20000 manifestants qui débordent complètement la police ${ }^{28}$. Celle-ci reconnaît début juin que la GLU contrôle efficacement toutes les principales industries de l'île.

$\mathrm{Au}$ moment de la libération de 1945, le PC, légal jusqu'en 1948, s'engage dans une politique de "Front Uni National", dans la perspective d'une décolonisation progressive, d'une unification de Singapour avec la Malaisie et de l'obtention d'une citoyenneté complète pour les Chinois et les Indiens, même nés en dehors du pays. Dans son programme en neuf points de 1946, les questions économiques n'occupent que les septième et huitième rangs. Les plus vastes mouvements sont directement politiques: grève générale du 30 janvier 1946, pour la libération d'anciens résistants arrêtés par les Britanniques; hartal (arrêt "gandhien" de l'ensemble des activités) en octobre 1947, en accord avec la Chambre de Commerce chinoise et les associations communautaires indiennes, pour protester contre la constitution d'une Fédération de Malaisie qui pérennise la séparation de Singapour aussi bien que les droits spéciaux des Malais, et reporte sine die les projets d'autonomie interne. Là encore, jusqu'à l'état d'urgence de juin 1948, qui renvoie les communistes à la jungle, le PCM paraît aller de succès en succès: seul parti organisé à l'échelle de la Malaisie entière, il contrôle des journaux, se rallie des petits mouvements politiques d'intellectuels anglophones (souvent eurasiens) et de Malais pro-indonésiens; et surtout la confédération syndicale qu'il contrôle, la Pan-Malayan Federation of Trade Unions (PMFTU) ${ }^{29}$ acquiert rapidement une hégémonie presque totale sur le mouvement ouvrier (les quatre cinquièmes des syndicats en font partie), en même temps qu'une puissance sans précédent: dès le 25 octobre 1945 un meeting réunit 20000 travailleurs à Singapour; fin 1947, à son apogée, elle peut revendiquer à l'échelle de la Malaisie 463 000 adhérents - à peu près autant que de salariés...

Un degré en dessous, c'est la même histoire qui se répète en 1954-56. La simultanéité de l'échec patent subi par la guérilla communiste et de l'accord du self-government par la nouvelle Constitution de 1954 permet un renouveau important des grèves (la plus dure touche en avril-mai1955 la compagnie d'autobus Hock Lee; on en compte au total 213 d'avril à septembre 1955, contre 13 pour 1953-54) et des syndicats, dont les adhérents passent de 76000 en 1954 à 139000 en 1955; le Singapore Trade Union Congress (STUC),

\footnotetext{
${ }^{27}$ Cf C.F. Yong, Tan Kah-kee: The making of an Overseas Chinese legend, Singapour, Oxford University Press, 1989

${ }^{28}$ SSPIJ, juin 1940

${ }^{29}$ Elle succède directement à la PMGLU, que les autorités avaient refusé d'enregistrer pour vices de forme
} 
qui en réunit la plupart, est progressivement conquis par les militants radicaux. Là encore l'unité se construit surtout sur le politique: perspective maintenant palpable de l'indépendance, soutien immédiat au People's Action Party (PAP), fondé en 1954, et dont les communistes clandestins espèrent faire leur cheval de Troie. L'autonomie revendicative des ouvriers est d'autant plus faible que les directions de leurs syndicats sont souvent tenues par des anciens lycéens chinois, exclus de leurs écoles lors de la dure répression anti-communiste de 1951-52, et par des étudiants indiens membres de l'influent Socialist Club de l'université. Ils vont devenir les chefs de file de l'aile gauche du PAP. Leur adhésion à la stratégie de front uni avec la direction modérée du parti, qui impose des concessions majeures, est renforcée, en 1956 par les quelque 300 arrestations qui décapitent les syndicats radicaux, et en 1957 par l'écrasante victoire du PAP aux municipales, qui laisse augurer de son succès électoral aux Législatives de 1959.

Les protagonistes sont largement les mêmes lors de ce champ du cygne (au moins provisoire) du mouvement ouvrier que sont les années 1961-63. La seule différence, c'est que les modérés du PAP, avec Lee Kuan Yew à leur tête, sont désormais au pouvoir, et que le front uni avec eux, qui paraissait toujours plus un marché de dupes tant les concessions étaient unilatérales, a été rompu en juillet 1961. L'aile gauche, contrainte de quitter le parti, a fondé le Barisan Sosialis (BS) ${ }^{30}$. Elle peut aussi s'appuyer sur les quelque 150000 adhérents de la Singapore Association of Trade Unions (SATU), héritière de $80 \%$ des effectifs de l'ex-STUC, le reste (essentiellement des cols blancs dans des secteurs fortement indianisés) ayant formé le National Trades Union Congress (NTUC) autour de Devan Naïr, transfuge du PCM, désormais "l'homme de Lee" dans le mouvement syndical. La répression frappe rapidement la SATU, dont plus de 300 cadres sont licenciés, dès les derniers mois de 1961: le mouvement de luttes sociales est loin d'atteindre l'ampleur de celui du milieu de la décennie précédente $(946$ 000 journées de grève en 1955, 454000 en 1956; 411000 en 1961, 165000 en 1962)31. Mais la stratégie suivie par les responsables radicaux est largement responsable de cette relative faiblesse: la scission avec le PAP prend pour justification la non libération de la totalité des détenus politiques, les restrictions en matière de libertés démocratiques, l'absence d'encouragement à l'unification du mouvement syndical et les quelques restrictions en matière de citoyenneté $^{32}$. La vraie raison est la mise en route d'un processus de fusion avec une Malaisie alors beaucoup plus répressive, et dont la composition ethnique ${ }^{33}$ interdit aux proches du PCM toute perspective de parvenir au pouvoir par les urnes.

Il est permis de penser que le BS s'est laissé ensuite piéger dans une stratégie électoraliste, qui échouera finalement aux Législatives de

\footnotetext{
${ }^{30}$ Front socialiste, en malais

${ }^{31}$ Margolin, op. cité, p. 306

${ }^{32}$ Ibid., p. 85

${ }^{33}$ Alors environ $50 \%$ de Malais, 33\% de Chinois, $15 \%$ d'Indo-Pakistanais
} 
septembre 1963: le parti, dont la plupart des dirigeants les plus compétents ont été emprisonnés en janvier, obtient le joli score de 33\% des voix, mais c'est insuffisant pour chasser le PAP du pouvoir. Il s'est très peu servi de son répondant dans le mouvement ouvrier: son secrétaire-général, Lim Chin Siong, avait dès l'origine appelé les travailleurs à "résister à toutes les tentatives de les provoquer à mener des actions militantes" et à "coopérer avec (les) employeurs là où de bonnes relations de travail existent déjà"34; un autre de ses dirigeants, Fong Swee Suan, va jusqu'à déplorer que le PAP ait "détérioré les relations entre le capital et le travail" 35 . Même lors des grèves, les revendications politiques seront très présentes. C'était ouvrir la voie au NTUC qui peut prétendre avec quelques arguments que "les procommunistes négligent l'intérêt des travailleurs", et, assez démagogiquement, que les "vrais syndicalistes" sont dans ses rangs ${ }^{36}$. Il est probable que la nature particulièrement composite de la base du BS constitua un handicap particulier par rapport aux précédentes périodes de luttes: non seulement les élèves des écoles chinoises y jouent toujours un rôle important, mais les squatters, dont la crise du logement a multiplié le nombre, sont devenus un facteur politique essentiel; enfin les intellectuels et fonctionnaires english-educated, souvent indiens, sont très présents parmi les cadres les plus en vue. Toujours est-il que c'est au moment où il est déja électoralement battu, sans stratégie de rechange, et au moment choisi par un pouvoir impitoyable pour attaquer la légalité même de la SATU et de ses principales composantes, que le BS se décide à abattre son joker syndical: une grève générale, les $8 / 9$ octobre 1963. Le mouvement est massif le premier jour: au moins 100000 grévistes. Mais la grève est décrété illégale, et 195 arrestations (dont le président de la SATU et deux autres députés nouvellement élus) décapitent le mouvement, qui s'effondre dès le 9. Dans le mois qui suit, sept des principaux syndicats sont désenregistrés (cf ci-dessous), ainsi que deux associations "rurales"37; le 12 novembre la SATU est dissoute. La voie est libre pour le NTUC, qui regroupe en décembre 1965 74\% des syndiqués - dont les effectifs ont diminué de $20 \%$ depuis $1962^{38}$.

Que la centralité des mots d'ordre politiques, ferments trop aisés d'unité, ait pu entraîner désorientation et finalement affaiblissement, on vient d'en voir un exemple. Les choses s'aggravent encore quand les mots d'ordre fluctuent, en fonction d'une conjoncture qui n'a rien à voir avec le mouvement ouvrier, et peu même avec le pays. De ce point de vue, les années d'avant-guerre sont exemplaires. Le PCM hésite entre trois stratégies au moins: le "classe contre classe" et l'anti-colonialisme radical, en particulier jusqu'au début de 1936; le "salut national" antijaponais, mais dans la dénonciation de la "trahison" du Kouomintang -

\footnotetext{
${ }^{34}$ Straits Times (Singapour, quotidien) -ST-, 19 juillet 1961

${ }^{35} S T, 14$ août 1961

${ }^{36}$ Devan Naïr, Communist Subversion of Trade Unions, document interne du PAP, non daté (antérieur à 1980), p. 95

${ }^{37}$ Il s'agit plutôt de "banlieusards", parfois agriculteurs

${ }^{38}$ Margolin, op. cité, pp. 112 et 306
} 
KMT- (y compris local) et des Britanniques; le salut national, en alliance avec les Chinois modérés et le colonisateur. Ainsi le congrès de Johore (septembre 1936) du PCM adopte la ligne du Front Uni avec le KMT, mais en traînant les pieds: le "salut national" n'est développé que parmi les intellectuels et étudiants ${ }^{39}$; en mars 1937 encore, le consulat de Chine à Singapour est dévasté par 300 ouvriers des biscuiteries (soutenus par la GLU), qui contestaient son rôle (passé) de médiateur ${ }^{40}$. Par contre, après l'agression du 7 juillet 1937, le PC décide de "se consacrer entièrement au mouvement de salut national anti-japonais, (et de) former un font uni avec tous les partis et courants", en jouant au maximum l'ouverture et la légalité, en particulier dans la presse ${ }^{41}$. Désormais les revendications ouvrières intégreront souvent le boycott des produits japonais, comme à Ho Hong Biscuit Factory où, en novembre 1937, on proteste contre les mauvais traitements dont les femmes sont victimes, et contre l'utilisation d'intrants nippons. En août 1939, tous les travailleurs du bâtiment donnent une journée de salaire à la Chine en guerre ${ }^{42}$. En mars 1938, les intitulés de l'ensemble des organisations liées au PC sont changés: la GLU devient ainsi Malayan Labouring Classes Anti-Enemy Backing-Up Society (MLCAEBUS), ce qui indique clairement le passage à l'arrière-plan de la dimension proprement syndicale. En décembre 1938, le PC, pourtant frappé par une répression renouvelée, déclare vouloir encourager l'amitié sinobritannique, s'abstenir désormais d'actions illégales et chercher le soutien des dirigeants traditionnels de la communauté chinoise; il se prononce pour un "front unique ouvrier, antifasciste, anti-guerre, anticapitaliste et anti-impérialiste" 43 - l'ordre des termes est significatif. La conférence du parti d'avril 1939 marque l'apogée de ce cycle de modération: on propose aux autorités coloniales de les soutenir en cas de guerre avec le Japon, à la seule coondition de l'accord des libertés fondamentales ${ }^{44}$.

Le pacte germano-soviétique marque un retournement important, mais pas au point de revenir au "classe contre classe" pur et simple; certes, en novembre 1939, le MLCAEBUS redevient la GLU; certes les grèves se développent à nouveau, qui dénoncent la "guerre impérialiste" et ses conséquences: "exploitation et suppression des libertés", inflation ${ }^{45}$; elles culminent au printemps de 1940 , et voient l'utilisation des méthodes violentes et des dénonciations ad hominem qui avaient marqué le mouvement anti-japonais. Mais c'est surtout la "trahison" de la Grande-Bretagne, le "Munich d'Extrême-Orient"46 qu'elle aurait

\footnotetext{
${ }^{39}$ SSPIJ, octobre 1936

${ }^{40}$ SSPIJ, avril 1937

${ }^{41}$ SSPIJ, juillet 1937

${ }^{42}$ SSPIJ, août 1939

${ }^{43}$ SSPIJ, décembre 1938

${ }^{44}$ SSPIJ, avril 1939

${ }^{45}$ SSPIJ, octobre 1939

${ }^{46}$ SSPIJ, septembre 1939
} 
conclu avec le Japon qui sont dénoncés ${ }^{47}$; de même, en juillet 1940 , le consul-général de Chine, la Chambre de Commerce chinoise et le KMT local sont attaqués pour leur refus d'ajouter aux actions anti-japonaises des actions anti-britanniques.

Le dernier retournement de la période suit de peu: dès août, le Comité Central reconnaît le manque d'écho de sa propagande contre la Grande-Bretagne ${ }^{48}$. En septembre, il prend acte du rapprochement qui vient de s'opérer en Chine entre $\mathrm{PCC}$ et KMT, et du raidissement des puissances anglo-saxonnes face à l'installation nipponne en Indochine française: tant les organisations à tonalité anti-britannique que les syndicats eux-même doivent mettre fin à leurs activités; il s'agit de former de nouvelles "organisations patriotiques" entièrement consacrées aux campagnes anti-japonaises ${ }^{49}$. En 1941, le PC offrira d'intégrer ses militants aux forces armées britanniques de Malaisie...

Les modalités de l'action: de l'unanimisme à la violence

Le mouvement prolétarien, souvent égaré sur les chemins périlleux de la politisation à outrance, voire de la manipulation sans vergogne par l'appareil communiste, a d'autant plus de mal à donner naissance à un authentique mouvement ouvrier que les syndicats qui en sont l'expression, depuis les années trente, sont fréquemment d'étranges créatures. Là encore on ne semble pouvoir échapper à la dualité corporatisme extrême/unanimisme araseur. Les premières associations légales de travailleurs étaient apparues à la suite de la Societies Ordinance de 1889. Elles sont pour l'essentiel le prolongement des guildes ancestrales transplantées de Chine: elles ne sont pas ouvertes aux non-Chinois (et correspondent souvent même à un seul groupe dialectal), elles mêlent généralement dans leurs rangs employeurs et salariés, elles correspondent à un métier étroitement défini. Des 175 "syndicats" présents à Singapour en 1939, seuls 49 ne s'adressent qu'aux salariés ${ }^{50}$; parmi ceux-ci, on compte aussi bon nombre d'amicales de cols blancs (comme la Clerical Union) ou de "bureaux de placement" pour la main d'oeuvre qualifiée, comme le Singapore Harbour Board Employees' Union (SHBEU) ou le Chinese Engineering Mechanics Association.

La stratégie des communistes fut de briser ces structures étroitement corporatives, voire catégorielles (en 1948 on comptait encore huit syndicats sur le port), en intégrant tant certaines des associations préexistantes que des individus dans des unions syndicales "fourre tout" 51 , invariablement étroitement centralisées et

${ }^{47}$ Pas tout à fait sans arguments: jusqu'à la défaite de la France, Londres entend donner la priorité à la guerre en Europe, en concédant au Japon la fermeture de la route de Birmanie et plusieurs accords commerciaux

${ }^{48}$ SSPIJ, août 1940

${ }^{49}$ SSPIJ, septembre 1940

50"Origins of unionism" (non signé), in Whos's who in the Labour Movement 1974-75, A. Ragunathan (ed.), sans date ni nom d'éditeur, p. 152

${ }^{51}$ Selon le rapport de 1948 des syndicalistes britanniques Awberry et Dalley (à utiliser avec précaution: ils étaient mandatés par les autorités coloniales, et se montrent très 
très politisées. Ce fut d'abord, avant-guerre, la GLU clandestine (cf cidessus). Entre 1945 et 1948, la SFTU profita de la légalisation et de l'importante latitude d'action offerte par la Trade Union Ordinance promulguée en 1940, mais appliquée en 1946 seulement. Il semble que la centralisation de la fédération ait été extrême: prélèvement de $10 \%$ des cotisations (le maximum légal) des adhérents, ordres de grèves et mots d'ordre revendicatifs impulsés d'en haut, toute puissance d'un appareil semi-clandestin qui apparaît peu dans le syndicalisme au quotidien ou dans les négociations.

On retrouvera dans les phases suivantes les mêmes traits fondamentaux. Ainsi, en 1954-56, un syndicat "généraliste", le Singapore Factory and Shop Workers' Union (SFSWU), se transforme, sous l'impulsion de militants radicaux issus pour la plupart du mouvement lycéen ou étudiant, en pivot d'un regroupement concurrent du STUC modéré (il est très proche des autorités), essentiellement indien et faiblement représentatif: le Middle Road Group, du nom de son lieu de réunion. Début 1956, le SFSWU compte une trentaine de milliers de syndiqués (un cinquième du total) répartis en une trentaine de sections locales ${ }^{52}$.

Après la dissolution des principaux syndicats de Middle Road, et l'emprisonnement de leurs dirigeants, le Singapore General Employees' Union (SGEU) va servir à son tour de pôle d'agrégation; en 1961, s'il n'est que l'un des 43 syndicats qui vont constituer le SATU, il regroupe un bon cinquième de ses quelque 150000 adhérents. Et, dans le processus de scission du PAP comme du STUC, le premier rôle revient aux mêmes Big 6, qui sont aussi dans une large mesure les anciennes figures de Middle Road... Un dernier essai du même type a lieu début 1964, autour du Singapore Commercial House and Factory Employees' Union, mais la répression l'arrête dans l'oeuf. La tradition "supraindustrielle" est si vive que, même dans le NTUC pro-PAP, deux syndicats non spécifiques (le Pioneer Industries Employees' Union, le Singapore Industrial Labour Organization) détiendront une primauté de fait, jusqu'à leur partage de 1980 en syndicats d'industrie.

L'inconvénient d'une telle tendance à la centralisation est double. D'une part les revendications tendent à s'éloigner des préoccupations concrètes des syndicats, à devenir très générales et très politiques - on en a vu des exemples. D'autre part la résistance à la répression s'affaiblit: une poignée d'arrestations sélectives à la tête (les Britanniques, puis le PAP y excelleront), et le mouvement entier s'effondre tel un château de cartes. Aurait-il pu cependant en aller autrement? Compte tenu de la taille de Singapour (1,6 million d'habitants en 1960), l'élite -y compris celle de la contestation radicaleest réduite. Par ailleurs l'irréductible diversité du prolétariat

anticommunistes), "la GLU, ancêtre des fédérations (membres de la PMFTU), intégra parmi ses membres toutes les catégories de travailleurs - tireurs de pousses et de cyclopousses, artisans, coolies de l'hévéa et de l'étain, coiffeurs, employés de bureau, hôtesses des dancings, filles de cabaret, et ainsi de suite", cité ibid. p. 155 52J-L Margolin, Singapour 1954-1980 - Economie, politique, société: une dialectique, thèse de 3ème cycle, université Paris-VII, 1982, p. 168 
singapourien imposait d'autres thèmes de regroupement que le discours de l'intérêt matériel.

Elle incitait aussi à d'autres moyens d'action que ceux, classiques, des ouvriers; ce d'autant plus que les pouvoirs successifs ont fortement eu tendance à considérer la moindre grève ou le plus petit meeting comme un acte de quasi-terrorisme, à réprimer en conséquence. Alors, tant qu'à subir la répression... Le petit nombre des militants et leur radicalisme spontané acheva de les pousser fréquemment à une violence organisée aux modalités souvent inspirées des sociétés secrètes chinoises, avec certaines desquelles des alliances tactiques furent d'ailleurs conclues, en particulier à la fin des années trente ${ }^{53}$. Ainsi le "picket corps" du PCM intervient-il dans les grandes grèves de 1936-47 en organisant des sabotages, ou en assassinant quelques contremaîtres et autres "traîtres"54. Reconverti dès juillet 1937 en Special Service Commission du mouvement de salut national entijaponais, il se fixe pour but "l'extermination des chiens courants". La violence se déchaîne souvent contre ceux qui brisent le boycott des produits japonais, qui emploient des Taïwanais "nipponisés", ou simplement qui refusent de "souscrire" pour la cause sacrée: menaces, bombes, vandalisme, parfois assassinats ${ }^{55}$. Des taxis transportant des Japonais sont attaqués; de jeunes Chinois contraignent même des Indiens à participer au boycott ${ }^{56}$. En juin 1940, la Special Branch britannique considère que le GLU a fait appel aux sociétés secrètes pour résister aux offensives policières contre les grèvistes ${ }^{57}$. Après la guerre, la conquête de l'hégémonie par le Singapore Federation of Trade Unions ${ }^{58}$ s'appuie parfois sur de rudes méthodes: ainsi, en septembre 1946, le président du SHBEU, sur le port, doit fuir Singapour, et son vice-président est frappé à plusieurs reprises; enfin, en octobre, ses locaux sont dévastés par de jeunes Chinois, qui s'empressent de constituer, en remplacement, une Singapore Harbour Labour Union ${ }^{59}$. Il semble que, dans les périodes suivantes, la violence régresse quelque peu, ou devienne un phénomène de masse, plus spontané, comme dans la grève des autobus de la compagnie Hock Lee (mai 1955), ou dans la riposte à la dissolution du syndicat des élèves des écoles chinoises (octobre 1956), qui dégénère en la plus grande émeute politique que Singapour ait connue.

\title{
Des occasions manquées?
}

Malgré ces insuffisances et ces dévoiements, un authentique mouvement ouvrier apparait clairement en gestation lors des grandes périodes de lutte. Les choses sont particulièrement nettes en 1936-37, avant l'éclatement de la guerre sino-japonaise: le PC n'est alors pas

\author{
${ }^{53}$ SSPIJ, août 1939 \\ ${ }^{54} \mathrm{Cf}$ en particulier SSPIJ, décembre 1937 \\ ${ }^{55}$ SSPIJ, décembre 1937 \\ 56Ibid., juillet 1938 \\ ${ }^{57}$ Ibid., juin 1940 \\ ${ }^{58}$ Section singapourienne du PMFTU \\ 59“Pre-war unionism"(sic - non signé), in Who's who... op. cité , pp 140-141
}


assez puissant pour tout contrôler, alors qu'il l'est suffisamment pour donner un minimum d'organisation interne et d'articulation externe aux mouvements sociaux; les échéances politiques apparaissent moins dramatiquement contraignantes qu'au cours des périodes suivantes. La combativité est forte, l'action presque unanime: ainsi les ouvriers du chantier de la base navale se massent-ils 24 heures durant devant le Protectorate of Chinese, avant d'être dispersés par la police ${ }^{60}$; ceux des usines d'ananas (qui dépendent de patrons chinois) mènent une action de longue durée, en essayant de porter le conflit en direction des plantations, dans le sultanat de Johore tout proche; la Singapore Traction Company connaît un mouvement spontané de deux jours et demi, que le PC aide au niveau du piquet de grève, etplusieurs des revendications sont satisfaites. Au total, en trois mois, on décompte une cinquantaine d'arrêts de travail ${ }^{61}$. La logique du déroulement de ceux-ci conduit les ouvriers à renforcer leurs syndicats: les nouveaux membres $\mathrm{y}$ affluent ${ }^{62}$, on va constituer des caisses de grève; les "syndicats jaunes" sont fréquemment éliminés. On vise à se passer des kangany-s et autres subcontractors, à instaurer un rapport salarial direct avec le patronat. Les revendications insistent souvent sur le refus des licenciements ainsi que sur le rattrapage du niveau des salaires de 1931, début de la crise; dans cette période de reprise économique, les demandes satisfaites sont assez nombreuses, et l'exemple est contagieux. Enfin, peut-être surtout, on s'adresse pour renforcer la lutte aux travailleurs d'autres entreprises, et les différences ethniques, dans le feu de l'action, s'atténuent singulièrement: des Tamouls, en particulier, adhèrent (souvent en groupe) aux syndicats, voire au PCM ce qui menace directement l'économie coloniale, du côté des plantations et des services publics, où les Indiens sont légion; ils vont constituer parmi les dirigeants du PMFTU un groupe bien plus nombreux que, comparativement, les Indiens ne le sont dans la population. Cet effritement des barrières entre communautés est peut-être ce qui effraie le plus le colonisateur: peu après le début de l'état d'urgence, en 1948, le président de la PMFTU, l'Indien Ganapathy, sera pendu.

Pourtant le mouvement syndical ne réussira jamais à se consolider, principalement du fait de l'interpolation dans son action d'échéances politiques des plus pressantes. Une fois de plus les années 1936-37 sont emblématiques. Dès le "double sept"63 qui marque l'invasion de la Chine, le PC et le GLU font du front inter-classiste qu'est le mouvement de salut national une priorité absolue. Les travailleurs indiens (dont, selon la police, 500 -essentiellement employés municipaux- venaient d'adhérer au parti64) ne sont évidemment guère concernés... Beaucoup d'étudiants, d'intellectuels,

${ }^{60}$ SSPIJ, septembre 1936

${ }^{61}$ Ibid., octobre 1936

${ }^{62}$ Ainsi les syndiqués seront, à Singapour, après de grandes vagues de luttes, 157000 en 1956 ou 189000 en 1962, contre 76000 environ en 1954, 129000 en 1958, 112000 en 1970

637 juillet 1937

${ }^{64}$ SSPIJ, juin 1936 
voire d'hommes d'affaires, et de nombreux prolétaires sont par contre gagnés (on a cité le cas exemplaire du milliardaire Tan Kah Kee), mais c'est sur la base du nationalisme, et la notion même de lutte de classes en est délégitimée. Il ne faut pas non plus négliger le poids de l'efficace répression britannique, qui pousse dans la même direction: pour se protéger tant bien que mal de l'arrestation, pour pouvoir s'exprimer dans la presse légale, la tentation est forte pour les militants de se "réfugier" dans un unanimisme ethnique, sous l'aile tutélaire d'organisations à peu près inattaquables, telles que la Chambre de Commerce chinoise.

Les années 1945-48 sont le theâtre d'une autre occasion manquée. Le contexte semblait encore plus favorable pour le PCM: légalisation et prestige exceptionnel apportés par son rôle éminent dans la résistance armée; popularité des références extérieures (1'URSS, le Parti Communiste Chinois); irruption à l'échelle régionale de la revendication de décolonisation; présence des travaillistes au pouvoir à Londres, pour la première fois; crise sociale très forte, alors que le caoutchouc et l'étain sont vitaux pour la balance des paiements britannique; quasi-absence de concurrents politiques crédibles, sauf chez les Malais en tout état de cause peu réceptifs ${ }^{65}$. Les syndicats prennent, on l'a vu, un essor sans précédent, et les Indiens y ont à nouveau une large place. Les grèves sont nombreuses, souvent très dures (quoique moins à Singapour qu'en Malaisie, où de nombreuses plantations sont occupées, avec parfois des tentatives d'autogestion). L'échec sera malgré tout encore au rendez-vous, pour trois raisons. Tout d'abord la répression coloniale est bien plus violente, plus sanglante surout qu'avant-guerre, en particulier sous la British Military Asministration (septembre 1945-avril 1946): dès le 14 février 1946, 24 responsables syndicaux sont arrêtés puis bannis et, le 15, 18 manifestants sont tués à Singapour et Johore Bahru. Par ailleurs le PC, répétant l'erreur d'appréciation de ses camarades vietnamiens en 1936, accorde au départ une importance exagérée au changement politique en métropole. Enfin et surtout, le parti privilégie 1"'anti-impérialisme" et la lutte pour l'égalité de toutes les ethnies, ce qui l'amène -par exemple lors du hartal du 20 octobre 1947- à s'unir au patronat chinois et indien ${ }^{66}$, dont ses membres syndiqués ont pourtant à subir de plein fouet la répression, lors des grèves. Ces alliés sont bien peu sûrs: dès le début de l'Emergency, la bourgeoisie chinoise se laissera massivement "retourner", et servira de support aux très pro-gouvernementaux Malayan Chinese Association et (à Singapour) Democratic Party.

Entre 1955 et 1963, le tableau est plus brouillé encore. Les luttes sociales sont plus sporadiques et se trouvent désormais coupées de l'hinterland malayen. Le PC est à nouveau interdit, la répression est presque constante, à l'exception, relative, du gouvernement David

\footnotetext{
${ }^{65}$ Malgré l'action non négligeable du Malay Nationalist Party, partisan de l'unification avec l'Indonésie, mais également proche du PCM ${ }^{66}$ Ils lancent à l'occasion des appels à l'intervention policière, en commun avec leurs collègues européens
} 
Marshall (1955-56) et des deux premières années du pouvoir PAP (1959-61). Les lycéens et étudiants chinois tiennent une place sans précédent, et font de la défense des études sinophones un enjeu politique de premier plan. La confusion de la ligne radicale est grande, au delà de la lutte largement consensuelle pour une décolonisation rapide: avec qui s'allier pour obtenir l'espace de liberté permettant la libération des détenus et l'expansion des syndicats? De quel nationalisme se revendiquer: le singapourien, le malayen ... ou le chinois, toujours sous-jacent? Les communistes soutiennent puis abandonnent le Labour Front de David Marshall, soutiennent puis abandonnent le PAP de Lee Kuan Yew, contribuent à fonder un Barisan Sosialis trop modéré quand il est puissant, trop radical quand il en a perdu les moyens. Le mouvement vers l'autonomie interne déclenché en 1954 par les Britanniques, celui pour l'indépendance qui prend vite le relais et galvanise les foules, l'accentuation du chômage et de la crise du logement, l'influence à son zénith des expériences révolutionnaires du tiers-monde (et d'abord de celle de la Chine communiste), la "trahison de classe" de certains membres de l'élite English-educated (par exemple le Socialist Club de l'université anglophone): tout cela ouvrait pourtant de vastes perspectives. Mais la colonisation avait su se trouver d'efficaces relais en Lee Kuan Yew et son équipe. Par ailleurs la coupure d'avec les gros bataillons ouvriers des plantations et des mines de la péninsule (si souvent à la tête des luttes les plus dures en 36-37 et 4548) constituait peut-être un élément de faiblesse irrémédiable.

\section{2 - Deuxième configuration: une classe ouvrière atone (1965-1995)}

Le surgissement de l'ouvrier moderne

Sur le tard (même par rapport aux autres "dragons"), à la fin des années soixante, Singapour accomplit enfin sa révolution industrielle. Non que, dans cet univers urbain, les ouvriers et assimilés ${ }^{67}$ aient augmenté leurs effectifs, qui au contraire ont fait preuve d'une remarquable stabilité: $39,6 \%$ en $1957,35,8 \%$ en $1976,38,8 \%$ en 1980 , $36,3 \%$ en $1985,34,3 \%$ en $1990,35,3 \%$ en 1994. Mais la part des travailleurs de l'industrie et surtout, dans celle-ci, de ceux employés dans les entreprises de dix salariés au moins a beaucoup cru: 16,3\% de salariés industriels en $1957,19,3 \%$ en $1966,26,9 \%$ en $1976,29,2 \%$ en 1980 (à la fin de la période de développement rapide des industries de main d'oeuvre); un tiers de ceux-ci environ (soit 5,5\% des actifs) seulement échappent aux micro-industries de moins de dix salariés en 1957, contre la moitié en 1966 (9,8\% des actifs), les neuf dixièmes en 1976 (24,3\%) et un peu plus encore en 1980 (27\%); quant aux ouvriers de l'industrie proprement dits (on ne dispose pas de données suffisantes les concernant avant 1979, mais ils représentent alors $80 \%$ de l'emploi manufacturier, et probablement au moins autant auparavant, compte tenu de la nature peu qualifiée de la plupart des activités), ils constituent en 1980 23,9\% des actifs. En valeur absolue,

${ }^{67} \mathrm{Cf}$ note 2 
la rapidité de l'industrialisation est plus impressionnante encore, compte tenu de la massivité de l'augmentation de la population active (fruit elle-même d'une croissance démographique supérieure à $3 \%$ jusqu'en 1960, et du passage du taux d'activité des 15-64 ans de 57\% en 1957 à 63,2\% en 1980), qui passe de 472000 en 1957 à 1093000 en 1980: les salariés de l'industrie bondissent de 77000 en 1957 à 108 000 en 1966, 232000 en 1976 et 312000 en 1980, soit un quintuplement en moins d'un quart de siècle; les ouvriers d'usine sont eux 255000 en 198068. Pour leur part, les salariés des entreprises manufacturières de dix employés au moins sont passés de 27400 en 1959 à 289000 en 1980 - soit plus qu'un décuplement.

Il s'agit là d'un cas où, pour paraphraser le vieil Engels, "la quantité se change en qualité": l'ouvrier d'industrie est devenu la figure habituelle du travailleur manuel (qui était plutôt auparavant le coolie "généraliste", engagé dans les services); le salarié de la moyenne ou de la grande entreprise est devenu la figure habituelle de l'ouvrier, jusque là marqué par un environnement essentiellement artisanal. Parmi les ateliers de dix employés au moins (les seuls comptabilisés), la moyenne se situait ainsi en 1959 à 48 salariés; en 1966 à 47; mais en 1976 à 83, en 1980 à $85^{69}$.

La plupart des caractéristiques de cette classe ouvrière enfin trouvée se trouvent profondément modifiées. La féminisation est massive: en 1957, 58000 hommes pour 17000 femmes dans l'industrie, soit 23\% de main d'oeuvre féminine; en 1980, 169000 hommes pour 144000 femmes, soit 46\% - exactement le double en pourcentage, et huit fois plus en nombre. On peut aller jusqu'à dire que le travail industriel est devenu une activité typiquement féminine: s'il occupait déjà en 1957 19,4\% des femmes pour 14,8\% des hommes (mais 76,6\% des hommes de 10 ans et plus avaient alors un emploi, contre 19,3\% seulement des femmes), en 1980 24,3\% des hommes, $38,5 \%$ des femmes s'y consacrent. On constate une adéquation remarquable entre l'industrialisation et la mise au travail des femmes, dont $44,3 \%$ des plus de 15 ans ont désormais un emploi en 1980, alors que, sous l'effet de l'allongement des études, le taux d'activité masculin n'a pratiquement pas changé (80,9\% en 1980).

L'immigration est elle aussi un phénomène majeur, quoiqu'assez mal connu faute de données précises quant à sa composition ethnique et à son insertion professionnelle. Il ne s'agit évidemment pas à Singapour d'un fait nouveau, mais plutôt d'une reprise assez massive,

${ }^{68}$ Sources: Economic Survey of Singapore, op. cité, différentes années; Ministry of Labour, Research and Statistics division, 1978 Singapore Yearbook of Labour Statistics, Singapour, 1979; You Poh Seng et al., "Population growth and population characteristics", in You Poh Seng \& Lim Chong Yah, The Singapore economy, Singapour, Eastern Universities Press, 1971; G. Shantakumar, "Human resources development in Singapore", in You Poh Seng \& Lim Chong Yah (ed.), Singapore: twenty-five years of development, Singapour, Nan Yang Xing Zhou Lianhe Zaobao, 1984

${ }^{69}$ Sources: Chia Siow Yue, "Growth and pattern of industrialization", in You \& Lim (1971), op. cité; Ministry of Labour, 1990 Singapore Yearbook of Labour statistics 
après une quasi-interruption, sous les coups des crises et des conflits, des années trente aux années soixante; par ailleurs il n'est pratiquement plus question pour les nouveaux immigrants d'obtenir la citoyenneté; enfin les principales sources traditionnelles -Chine et Indeont été relayées par d'autres. On estime qu'en 1973 travaillaient à Singapour quelque 100000 étrangers (soit plus de 10\% des actifs), alors presque exclusivement des Malaysiens d'ethnie chinoise. Beaucoup sont renvoyés lors du "premier choc pétrolier", ce qui permet une augmentation du chômage limitée à $0,5 \%$ des actifs. Avec la forte reprise de la fin de la décennie, ils sont à nouveau plus d'une centaine de milliers en 1980 (105000 disposent cette année-là d'un permis de travail salarié, y compris les "expat" venant des pays avancés) ${ }^{70}$, avec une proportion croissante de Thaïlandais, de Srilankais et de Bangladais. Bon nombre seront expulsés lors de la crise de 1985-86. Au début des années quatre-vingt dix, les travailleurs immigrés sont estimés aux alentours de 250 000, soit $15 \%$ au moins des actifs. Une part importante des étrangers est employée dans le bâtiment et les travaux publics (où ils sont sans doute depuis longtemps très majoritaires), et de plus en plus dans les services (domesticité en particulier); ils ont néanmoins toujours été surreprésentés dans l'industrie, et surtout dans les activités de main d'oeuvre peu qualifiée.

Il est vrai que celles-ci ont été au coeur de la première phase (jusque vers 1980) du processus d'industrialisation. En 1959, les trois branches principales étaient l'agro-alimentaire $(21 \%$ de la main d'oeuvre industrielle), le papier et l'édition (15\%) et les machines et engins (10\%). En 1980, ce sont les "machines" -pour l'essentiel l'électronique- (38\%), le textile et la confection (15\%) et l'équipement de transport (11\%) - qui correspond surtout à la construction de navires et de plate-formes pétrolières. Les "machines" sont passées de moins de 3 000 salariés à 111 000, le textile d'un millier à 40000 ! Par contre le raffinage pétrolier, activité plus qualifiée, n'est passé que de 1000 à 4 000 employés, le papier-édition de 4000 à $16000^{71}$. Cela rend compte d'une forte tendance à la stagnation, voire la baisse des salaires tout au long de la période, seule la régression du chômage et du nombre d'enfants à charge permettant une augmentation du revenu disponible par ménage. Un travailleur des constructions électriques touchait en moyenne mensuelle $190 \mathrm{~S} \$ 7^{72}$ en 1959, $247 \mathrm{~S} \$$ en 1963 (mais il s'agissait alors surtout d'ouvriers qualifiés masculins); les six ans qui suivent, la baisse en nominal (il faudrait y ajouter une inflation totale d'environ $7 \%$ ) est très forte, et correspond à la phase de féminisation et de déqualification (il s'agit désormais d'assemblage électronique simple): 232 S\$ en 1965, 124 S\$ en 1969; en 1978 on est remonté à 206 S\$ mais, compte tenu d'une hausse des prix d'environ $80 \%$, cela signifie une stagnation au bas niveau de 1969, alors que la semaine de travail

\footnotetext{
${ }^{70}$ Chiew Seen Kong \& Ko Yiu Chung, "The economic dimension", in Stella R. Quah et al., Social Class in Singapore, Singapour, Times Academic Press, 1991, p. 119

${ }^{71}$ Margolin, op. cité, annexe I d, p. 296-97

${ }^{72} \mathrm{Le}$ dollar de Singapour vaut généralement autour de 4FF
} 
reste supérieure à 48 heures. Simultanément un ajusteur (masculin) des chantiers navals gagne $411 \mathrm{~S} \$$, un mécanicien $472 \mathrm{~S} \$$... $^{73}$

Le dernier caractère essentiel de la nouvelle classe ouvrière singapourienne est sa dépendance vis-à-vis de l'international. Celle-ci est double. D'une part, dès 1966, le choix est fait par les dirigeants du PAP du passage d'une industrialisation par substitution d'importations (rendue impraticable par la séparation d'avec la Malaysia, en août 1965) à la promotion des exportations manufacturées: le petit marché local, jusque là raison d'être des faibles industries singapouriennes (il représentait encore 56\% de leurs débouchés en 1970) n'absorbe plus en 1980 que le quart de leurs ventes, qui s'étendent au monde entier (singulièrement à l'Amérique du Nord et à l'Europe), et participent largement au seul commerce extérieur exportant plus que le PIB du pays (compte tenu des réexportations): 6,2\% de produits locaux dans les ventes en 1960, 35,9\% en $1968,62,3 \%$ en $1980^{74}$. D'autre part l'investissement industriel devient le privilège presque exclusif des sociétés multinationales: 85\% du total en 1976 comme en 1980, 81\% en $1986,89 \%$ en $1990,75 \%$ en 1994 . Dans les seules années $1968-74$, la main d'oeuvre des établissements manufacturiers à capitaux étrangers à $100 \%$ passe de $12 \%$ à $36 \%$ du total; celle des usines à capitaux locaux à 100\% régresse de 59\% à 30\% ${ }^{75}$ - la balance étant à mettre au compte des nombreuses joint-ventures, dans lesquelles, à cette époque surtout, le management est généralement aux mains des cadres étrangers, mieux qualifiés. Depuis un quart de siècle, Singapour a sans doute l'appareil industriel le plus extraverti qui soit; seul un petit nombre de sociétés publiques, pour la plupart liées au ministère de la Défense, ou spécialisées dans la construction navale, se sont imposées à côté des filiales des grandes multinationales.

Les sources endogènes de la paralysie ouvrière

On trouve dans plusieurs des caractères fondamentaux de la nouvelle classe ouvrière singapourienne des raisons (sinon toutes les raisons) à son atonie. Certes le progrès de la concentration industrielle, joint à l'inflation des effectifs, fournit en théorie des moyens d'action non négligeables, et la base d'une autonomie, jamais réalisée durablement auparavant, par rapport au "vieux" prolétariat des services, du commerce, ou par rapport aux étudiants. La quasistagnation des plus bas salaires jusque vers $1980^{76}$, alors que la croissance annuelle du PIB dépasse fréquemment les $10 \%$, et que les fonds dégagés pour l'investissement (FBCF) passent de 8,4\% du PIB en 1960 à 32,2\% en 1970 et 42,2\% en 1980 (34,5\% en 1994), aurait sans doute dû depuis longtemps servir de combustible à de fortes

\footnotetext{
${ }^{73}$ Ibid., annexe VII, p. 307, et Margolin (1982), op. cité, pp. 624-25

${ }^{74}$ Margolin (1989), op. cité, annexe I Ea, pp. 298-99

${ }^{75}$ Lim Joo-jock, Foreign investment in Singapore, Singapour, ISEAS, 1977, pp. 126127

${ }^{76}$ Malgré un doublement approximatif (inflation une fois déduite) du salaire moyen entre 1960 et 1980 suivant des données officielles, mais contestables- cf Margolin (1989), p.250 pour une discussion précise
} 
revendications siciales. On verra plus loin que le risque fut d'ailleurs pris très au sérieux par les autorités, qui s'ingénièrent à entraver nouveaux et anciens travailleurs de mille liens redondants. Néanmoins, pour paraphraser Abraham Lincoln, on ne peut réprimer tout le monde (et pas même quelques centaines de milliers d'ouvriers) tout le temps (et sans doute pas même un quart de siècle): de la Pologne à la Corée du Sud, les exemples contemporains de révoltes ouvrières déclenchées dans les pires conditions ne manquent pas, y compris dans les pays de la fameuse orbe confucéenne. Il convient donc de s'interroger d'abord sur la sociologie de la résignation ouvrière.

Une place essentielle y est jouée par le changement profond de population des ateliers. A la fin des années cinquante, elle était très masculine, presque exclusivement autochtone (si l'on adjoint aux localborn les immigrants depuis longtemps stabilisés, et naturalisés) et relativement qualifiée, au moins par rapport à la masse du prolétariat. Aux alentours de 1980, elle est majoritairement composée de femmes et d'immigrés ${ }^{77}$. Or ces deux catégories constituent, pour des raisons partiellement similaires, une main d'oeuvre malléable, et peu apte à revendiquer. Il s'agit d'ouvriers à titre précaire, pendant une étape de leur vie: en 1978, si 41,4\% des jeunes filles de 15 à 19 ans travaillent, si leur nombre monte à $73,2 \%$ entre 20 et 24 ans, elles ne sont plus que $53,1 \%$ dès $25-29$ ans, et $36,8 \%$ entre 30 et 34 ans; le taux ne cesse de décroître ensuite, sur un rythme plus lent; la majorité des femmes salariées sont donc très jeunes (68\% ont entre 15 et 29 ans), et ne le sont que pour 10 à 15 années de leur vie ${ }^{78}$. Quant aux travailleurs étrangers ${ }^{79}$, ils ne disposent généralement que d'un contrat de trois ans, en principe non renouvelable, et tout est fait -non accession aux logements sociaux, interdiction de se marier ou d'avoir des enfants, résidence forcée en Malaisie pour des dizaines de milliers d'entre euxpour les empêcher de considérer leur situation comme stable. Les femmes sont par ailleurs victimes du "syndrôme Confucius", qui les pousse à privilégier leur fonction filiale, sororale ou maternelle (démissionner à la première grossesse constitua longtemps une sorte de règle non écrite), et à considérer en conséquence leur vie professionnelle comme accessoire ${ }^{80}$, leur paye étant réduite au rang de salaire d'appoint, versé dans le pot commun familial. Les immigrés, eux, sont

${ }^{77} \mathrm{Il}$ n'est pas facile de risquer un chiffre: seule la main d'oeuvre de l'industrie (pas les ouvriers proprement dits) est décomposée suivant le sexe (46\% de femmes en 1980), et les $11,3 \%$ de travailleurs immigrés légaux ne sont pas précisément répartis professionnellement; sachant cependant qu'en dehors du bâtiment ils travaillent surtout dans l'industrie, on peut estimer que femmes plus immigrés (ce sont assez souvent des immigrées...) représentent au strict minimum une grosse moitié des ouvriers

${ }^{78}$ Margolin (1982), p. 624

${ }^{79} \mathrm{Qu}$ 'il faut distinguer des nombreux “expatriés” qualifiés, issus des pays avancés, dont la venue est au contraire encouragée

${ }^{80}$ D'où le refus très courant du mariage et de l'enfantement chez les femmes diplômées, désireuses, elles, de réussir - ce qui préoccupa beaucoup Lee Kuan Yew au milieu des années quatre-vingt: comme quoi il est difficile d'avoir la sauce de soja et l'argent de la sauce de soja 
des personae non gratae par principe, systématiquement marginalisées (jusque dans les statistiques, étrangement déficientes en ce qui les concerne), vivant principalement dans des baraquements de chantier ou des foyers, interdits d'association, de responsabilité syndicale, de vie familiale, pieds et poings liés à leur employeur qui les fait rentrer dans le pays, et qu'ils ne peuvent quitter sous peine d'expulsion immédiate.

La rupture avec la situation qui prévalait jusque dans les années cinquante est fondamentale: les China- et India-born avaient alors la quasi-certitude de pouvoir s'intégrer à la société singapourienne, sans subir de discrimination juridique (sinon, dans de rares cas, l'expulsion pour gangstérisme, appartenance à une société secrète ou "subversion" politique). On agite de temps à autre le gros bâton: annonce en 1989 de mesures contre l'immigration illégale, dont la plus spectaculaire est le flogging (bastonnade judiciaire) des contrevenants ${ }^{81}$; exécutions assez fréquentes de petits trafiquants de drogue, pour la plupart malaysiens; peines très sévères pour les immigrés délinquants, avec lesquels la justice singapourienne se montre encore plus impitoyable que d'ordinaire 82 . Les syndicats ne sont d'aucune aide, bien au contraire: en juillet 1979, Devan Nair, président du NTUC, fixe pour objectif à l'action syndicale de "remplacer le présent flux sans cesse croissant de travailleurs étrangers par un reflux constant de ces mêmes travailleurs dans la direction opposée"83. On constate d'ailleurs une syndicalisation presque nulle dans le bâtiment, où les immigrés sont le plus concentrés: 703 syndiqués sur 134000 salariés en $1990 !^{84}$.

L'amélioration, ou la perspective d'amélioration des conditions de vie, pour soi ou pour ses descendants, est un puissant anesthésiant. On ne répétera jamais assez que les NPI extrême-orientaux, s'ils ont longtemps contenu les salaires dans d'étroites limites, n'ont jamais diminué les revenus de catégories significatives de leurs populations; compte tenu de la rapidité de la transition démographique, de la quasidisparition du chômage, de la mise au travail massive des femmes, le développement accéléré signifia dès l'abord, et constamment ensuite, un peu plus de bien-être, non pour dans vingt ans, mais immédiatement. Néanmoins, jusqu'aux alentours de 1980, on l'a vu, les salaires réels stagnèrent souvent à Singapour. Ce n'est plus le cas ensuite, alors même que les possibilités de gains indirects susmentionnés s'épuisent. Si l'on suit les statistiques officielles ${ }^{85}$, le salaire mensuel moyen réel des production, transport and other manual workers a augmenté de 73\% entre 1980 et 1990; dans l'industrie, la

\footnotetext{
${ }^{81}$ Cela provoqua une vive tension avec la Thaïlande, dont des dizaines de milliers de ressortissants étaient visés; elle organisa leur exode à l'aide de sa flotte de guerre ${ }^{82}$ D'où, en mars 1995, l'énorme mobilisation de la population et des autorités des Philippines en faveur de la femme de ménage Flor Contemplacion, pourtant reconnue coupable du meurtre de ses patrons singapouriens, et finalement exécutée.

${ }^{83}$ Perjuangan, vol. $3, \mathrm{n}^{\circ} 7$, juillet 1979

${ }^{84}$ Singapore Yearbook of Labour Statistics, op. cité, p. 53

${ }^{85}$ Elles ne sont pas entièrement compatibles entre elles en matière de rémunérations, ce qui incite à les considérer avec prudence, mais ne remet pas en cause la direction et l'ampleur de l'évolution
} 
progression serait même de $78,9 \%$, dans le bâtiment de $99,5 \%{ }^{86}$. Cela ne représente pas encore des rémunérations particulièrement élevées, et l'éventail hiérarchique est extrêmement ouvert, en particulier entre professions féminines peu qualifiées et professions masculines qualifiées: les assembleuses d'électronique (certainement la profession la plus nombreuse) ont pour salaire médian 531 S\$ en 1990, les employées de boutique 630 S\$, les dactylos 820 S\$, les employées de bureau $828 \mathrm{~S} \$$; les salaires des manoeuvres masculins sont répartis autour de 640 S\$, ceux des mécaniciens de l'industrie autour de 1000 $\mathrm{S} \$$, ceux des contremaîtres de l'électronique autour de $1445 \quad \mathbf{S} \$ 87$. Néanmoins, compte tenu du faible coût des logements sociaux, de l'école (du moins par rapport aux pays d'Asie du Nord-Est) et de l'alimentation quotidienne, cela suffit à assurer à la plupart une existence point trop médiocre, et en tout cas à l'abri des besoins élémentaires. De plus les fonds prélevés en vue de la retraite $(35 \%$ à $40 \%$ des salaires ces dernières années) diminuent l'incertitude de l'avenir, et, pouvant être partiellement mobilisés pour l'achat d'un appartement, ont assuré le succès d'une politique "à la Chalandon", non dénuée d'arrière-pensées politiques: plus de $60 \%$ des Singapouriens sont désormais propriétaires.

Mais les mutations rapides de la société singapourienne donnent plus encore d'espérances que de satisfactions. $63,8 \%$ des habitants de la cité-Etat s'estiment en meilleure position que leurs parents sur le plan économique, contre $12,7 \%$ d'un avis contraire; $52,8 \%$, contre $6,1 \%$, pensent que l'amélioration se poursuivra avec leurs enfants ${ }^{88}$. $81 \%$ se situent eux-mêmes dans la "classe moyenne" (dont $44 \%$ dans la "moyenne moyenne"), contre $17 \%$ seulement dans la lower class, et $1 \%$ dans l'upper ${ }^{9}$. La réalité appuie pour une part ce parti-pris d'optimisme. On constate en effet une assez forte mobilité sociale: ainsi la cohorte des 20-29 ans de 1957 comprenait $82,3 \%$ de salariés, et $1,6 \%$ d'employeurs; la même cohorte, en 1986 , compte $63,8 \%$ des premiers, mais $11,9 \%$ des seconds ${ }^{90}$. La cohorte des 20-29 ans de 1966 compte $11,8 \%$ de professionals, technicians \& managers; elle en comprend $19,2 \%$ en $1976,20,5 \%$ (à 40-49 ans) en 1986; chez les 40-49 ans, on ne comptait en 1976 que $14,9 \%$ de ces derniers, et que $8,2 \%$ en $1966^{91}$.. La dynamique du développement se conjugue avec l'extension de la formation initiale pour expliquer le phénomène: en 1986, 60\% environ des actifs diplômés du supérieur ont moins de 40 ans $^{92}$.

Tout n'est pourtant pas rose dans l'évolution économique, tant s'en faut. Ainsi les travailleurs manuels non qualifiés n'oscillent qu'entre $35,6 \%$ et $35,9 \%$ dans la cohorte considérée. On a constaté

861990 Singapore Yearbook of Labour Statistics, op. cité, p. 26

${ }^{87}$ Ibid. pp. 27 à 30

${ }^{88}$ Chiew \& Ko, art. cité, pp. 126-129

${ }^{89}$ Sharon Mengchee Lee, "Social class in Singapore: an overview", in Quah (ed.), op. cité, p. 27

${ }^{90}$ Chiew Seen Kong, "Social mobility in Singapore", in Quah (ed.), op. cité, p. 192

91 Ibid. pp. 203-205

${ }^{92}$ Ibid. p. 200 
l'importance des inégalités salariales; celle des revenus, pris globalement, ne fait que la confirmer: en 1984, le quart le plus pauvre des actifs ne reçoit que $5 \%$ des revenus; les $5 \%$ les plus riches en accaparent le quart ${ }^{93}$. Enfin le "méritocratisme" cher aux dirigeants de la cité-Etat, et auquel nombre de leurs concitoyens adhèrent fortement, ne semble pas entièrement corroboré par l'analyse des trajectoires professionnelles réussies: la réussite scolaire n'en serait l'explication principale que dans un cas sur quatre environ, les connexions familiales ou personnelles et l'entregent tenant une place au moins aussi importante ${ }^{94}$. De cela les Singapouriens sont, pour une part, conscients: $61 \%$ (contre 35,7\%) sont d'accord avec l'affirmation: "les pauvres ont très peu de chances d'être entendus sur les questions qui intéressent la société"; 53\% (contre 43,5\%) estiment que "les riches peuvent se sortir d'à peu près n'importe quoi grâce à leur argent"; et il est frappant de constater, sur ces opinions, le faible écart entre les réponses de ceux qui se jugent "réussis" ou "ratés"95. Il n'empêche: ce sont là des contradictions pour l'instant relativement secondaires, et qui, manifestement, à en juger à l'aune des résultats électoraux et de la paix sociale, ne suffisent à remettre en cause ni le mouvement ascensionnel de la société singapourienne, ni la confiance qu'une bonne partie des plus défavorisés eux-mêmes manifestent dans la règle du jeu, et dans leur propre aptitude (ou dans celle de leurs enfants) à en jouer avec sagacité.

Les causes exogènes: mutation démographique, contrainte politique

La population singapourienne, dont un tiers à peine était née dans l'île au début des années trente, a connu, du fait du blocage progressif de l'immigration à partir de 1931, un rapide mouvement de localisation : les local-born constituent depuis 1980 plus des quatre cinquièmes des Singapouriens - et beaucoup plus encore des moins de 40 ans. Mais cet éloignement progressif d'avec les terres d'origine a été redoublé dans le cas de la Chine par une rupture des relations à peu près complète, une trentaine d'années durant, à la suite de la révolution de 1949. Enfin le régime communiste chinois, après avoir beaucoup séduit, a beaucoup déçu. Le nationalisme et le communisme chinois, transitoirement associés, de 1937 aux années soixante, dans l'esprit de beaucoup de Singapouriens, ont représenté une redoutable force propulsive. Son élan paraît désormais brisé, sans recours. Compte tenu des impasses auxquelles mena cette combinatoire (cf ci-dessus), son affaiblissement aurait pu conduire à un mouvement ouvrier rénové, et plus efficace, sur une base davantage multiethnique, ou même singapourienne, tout simplement. On sait qu'il n'en fut rien, du fait des mutations socio-économiques concommitentes, du fait aussi de l'incapacité de l'opposition de gauche politico-syndicale à sortir d'une

\footnotetext{
${ }^{93}$ Chiew \& Ko, art. cité, p. 131

${ }^{94}$ Ko Yiu Chung, "Status Attainment", in Quah (ed.), op. cité, pp. 231-236

${ }^{95}$ Chiew Seen Kong, Ko Yiu Chung \& Stella R. Quah, "Occupational prestige and occupational structure", in Quah (ed.), op. cité, pp. 97-99
} 
configuration idéologique surranée, qu'elle ressassa jusqu'à son progressif effacement.

Du fait enfin, bien entendu, de la dure répression que subirent les militants actifs, et de l'enserrement des ouvriers dans une forêt de lois et de structures les privant de presque toute possibilité d'action autonome. L'évolution du droit syndical et de la réglementation des relations du travail est de ce point de vue exemplaire. Non que l'héritage de la période coloniale ait été particulièrement libéral: la Trade Unions Ordinance de 1940 (appliquée, du fait de la guerre, en 1946 seulement), premier texte officiel à reconnaître la légitimité des syndicats ouvriers, vise aussi à limiter leur liberté d'organisation sur de nombreux points, et surtout leur impose une procédure -non automatiqued'enregistrement, longue et complexe, qui assure de fait au pouvoir exécutif de larges possibilités de contrôle et de pressions, dont il usera avec de plus en plus de libéralité ${ }^{96}$. Un amendement, en 1948, dans le contexte de l'Etat d'urgence et de l'affrontement armé avec les maquis communistes, impose le vote à bulletins secrets de toute décision importante, ouvre les livres de compte et la liste des membres au Registrar of Trade Unions, et lui permet d'empêcher toute utilisation "illégale" des fonds ${ }^{97}$.

Mais le PAP "socialiste" - et jusqu'en 1976 membre de l'Internationale du même nom- aggravera de plus en plus cette situation, et conduira les syndicats à un véritable enfermement juridique. Faisant mine de lutter contre les syndicats jaunes, scissionistes ou étroitement catégoriels, et de répondre ainsi aux revendications de sa base électorale très à gauche, il fait adopter dès 1959 un amendement accordant au Registrar une grande latitude pour refuser d'enregistrer ou désenregistrer tout syndicat "allant contre l'intérêt des travailleurs", même s'il satisfait à toutes les obligations légales; au nom de cette notion floue, évidemment non définie (on la fera par exemple jouer pour arbitrer le conflit de deux syndicats concurrents dans une même branche), le gouvernement de Lee Kuan Yew dissoudra 61 syndicats entre 1959 et $1966^{98}$, y compris, en 1963, le "corps de bataille" de la SATU (en fait les plus grandes organisations ouvrières du moment), et cette confédération elle-même. De plus l'appel contre une décision du Registrar remonte au ministre du Travail (qui n'est même pas obligé de motiver son avis), sans possibilité ultérieure de recours judiciaire.

Une Trade Unions Bill, votée en 1960, réduit ces pouvoirs quasidiscrétionnaires, affirme le principe de la démocratie interne des syndicats, leur accorde le droit à l'action politique, et impose des sanctions aux employeurs non respectueux du droit d'association. Mais cette loi, qui fait la part trop belle aux revendications des procommunistes, ne sera jamais promulguée. Par ailleurs 1960 est marqué

\footnotetext{
96Venkatraman Anantaraman, Singapore industrial relations system, Singapore Institute of Management/McGraw-Hill Book Co, 1990, p. 101

${ }^{97}$ Ibid. p. 102

${ }^{98} \mathrm{Ibid}$, p. 108
} 
par l'entrée en vigueur de l'arbitrage obligatoire des conflits du travail (prévu dans le programme électoral de 1959), destiné à limiter le recours à la grève; Devan Nair, qui dirige alors la fraction modérée des syndicats, fut plus tard très clair sur son sens: "Manquant du soutien syndical dont bénéficiait le front uni communiste, la direction du PAP tenta de neutraliser le potentiel politique des syndicats (...) L'Industrial Arbitration Court (IAC) fut mise en place et le ministre du Travail reçut le pouvoir d'ordonner l'arbitrage des conflits" 99

Dès l'année suivante, la scission du PAP fait définitivement pencher la balance dans le sens du corsetage de l'autonomie ouvrière. Un amendement de 1966 au Trade Unions Act interdit aux noncitoyens et aux syndicalistes ayant subi une condamnation toute fonction de responsabilité, et impose le contrôle du Registrar sur le vote à bulletins secrets indispensable pour toute "action industrielle", dont la définition est par ailleurs étendue bien au delà de la seule grève. Les coups portés en 1968 -au moment où les multinationales commencent à affluer- sont bien plus accablants, et constituent aujourd'hui encore la clé de voûte de cette "paix du travail" (industrial peace) que le gouvernement présente comme une manière d'impératif catégorique ${ }^{100}$. L'Industrial Relations Act (IRA) confère à l'employeur pleine autorité pour décider des méthodes de production et des relations du travail, et plus précisément de l'embauche (et la réembauche), la mise à pied, le licenciement, la promotion, le transfert, l'affectation interne. Toute action syndicale sur ces divers points, quelle qu'en soit la forme, et même si la décision patronale était condamnable sur le fond, serait donc automatiquement illégale. Seul un recours devant l'IAC peut, dans certains cas très limités (comme le licenciement systématique de syndicalistes, mais pas le choix arbitraire des licenciés), ordonner le réemploi d'un travailleur; dans les autres cas, il est possible d'en appeler au ministre du Travail, qui a toute latitude de confirmer un licenciement même si la procédure légale n'a pas été respectée, d'organiser ou non une enquête, et dont la décision est définitive - la rapidité de celle-ci étant présentée comme justifiant son caractère largement arbitraire ${ }^{101}$.

La même année, l'Employment Act uniformise, par le bas, des avantages et conditions d'emploi qui pouvaient varier considérablement d'une branche à l'autre et dont, suivant le modèle britannique, l'essentiel des dispositions était laissé à la libre négociation entre employeurs et salariés. Il s'agit aussi de relier au maximum la rémunération à la qualification, à la productivité et aux résultats de l'entreprise - obsession technocratique dont on verra qu'elle a du mal à s'imposer. La loi vise à réduire de nombreux avantages sociaux, en

${ }^{99}$ Devan Naïr, Communist subversion of Trade Unions, document pour les cadres du PAP, 1978

100"Notre force économique s'accroîtra aussi longtemps que le gouvernement, les travailleurs et les chefs d'entreprise tireront dans le même sens, avec le minimum de frictions et le maximum d'esprit de réalisme et de conciliation": Lee Kuan Yew, in Alex Josey, Lee Kuan Yew, Singapour, Asia-Pacific Press, 1971, p. 532

${ }^{101}$ Anantaraman, op. cité, pp. 127-131 
particulier ceux des cols blancs: passage dans tous les secteurs de la semaine de travail à 44 heures; réduction des jours fériés de 15 à 11 , des congés-maladie payés de 28 à 14 jours; congés payés limités à deux semaines, après dix ans de stabilité d'emploi; limitation à 100\% au maximum (200\% auparavant) de la prime pour heures supplémentaires. Il convient enfin de faire régner l'ordre dans l'entreprise, et de pénaliser l'infidélité au patron: licenciement immédiat au bout de deux jours d'absence non justifiée; remboursement des intrants détériorés par la faute du travailleur; suppression de l'indemnité de licenciement si l'emploi a été occupé moins de trois ans. Enfin, pour faciliter la gestion et limiter les hausses de salaires, la durée des contrats collectifs passe de 18 mois-3 ans à 3ans-5ans ${ }^{102}$; l'IAC reçoit mission de certifier la conformité à la loi de ces contrats, qui acquièrent ainsi la valeur de décisions de justice.

Le troisième des piliers légaux régissant les relations du travail est mis en place en 1972, avec l'entrée en fonction du National Wages Council (NWC). Cet organisme tripartite, qui entend symboliser la collaboration du patronat, des salariés et de l'Etat sur l'autel de la productivité, est en principe seulement consultatif. Mais ses avis, et surtout ses recommandations annuelles concernant le montant et les modalités de l'évolution des rémunérations, sont à peu près systématiquement pris pour base des accords d'entreprise et, en cas de contestation, des décisions de l'IAC. Jusqu'en 1979, il s'agit tout à fait explicitement d'empêcher les salaires de croître plus vite que la productivité, alors même que la réalisation progressive du plein emploi devrait, dans les conditions d'un libre marché du travail, les tirer fortement vers le haut. En 1977, le NWC prétend même supprimer toute augmentation aux salariés "déméritants", que ce soit par négligence ou par incapacité, et à ceux présents dans l'entreprise depuis moins d'un an ${ }^{103}$. En 1981, il organise la fourniture à l'employeur de l'histoire professionnelle récente (trois années) d'un candidat à l'embauche.

Les quelques failles légales par lesquelles la contestation ouvrière aurait encore pu s'engouffrer sont comblées l'une après l'autre. Ainsi un amendement de 1977 étend des responsables syndicaux à l'ensemble des salariés la possibilité de poursuites judiciaires pour incitation ou même participation à une action illégale. Un autre, la même année, étend encore le contrôle des fonds du syndicats par le ministre du Travail: depuis 1963, il pouvait en geler l'utilisation en cas de procédure de désenregistrement; désormais une simple investigation, fiscale, judiciaire ou du Registrar aura le même effet. Ce dernier, en 1982, reçoit de larges pouvoirs de contrôle sur la comptabilité des syndicats. Simultanément, un nouvel amendement au Trade Unions Act établit comme motif de refus d'enregistrement ou de désenregistrement d'un syndicat toute clause "oppressive or unreasonable" figurant dans ses statuts; il s'agissait de réagir au

102Margolin (1982), op. cité, pp. 606-608

${ }^{103}$ Anantaraman, op. cité, pp. 125-126 
mauvais exemple donné par les United Workers of Petroleum Industry (affiliés au NTUC) qui prétendaient chasser de leurs rangs les partisans d'un éclatement en syndicats d'entreprise, désormais recommandés par le pouvoir (cf ci-dessous). Par ailleurs, sans que la mention du droit de grève disparaisse de la législation, la nouvelle définition de l'objet du syndicat de travailleurs incluse dans l'amendement la fait disparaître, au profit de la "promotion de bonnes relations entre travailleurs et employeurs" ainsi que de la "réalisation de gains de productivité à l'avantage des travailleurs, des employeurs et de l'économie de Singapour", alors que le texte de 1940 évoquait "le lancement, l'organisation ou le financement des grèves"104. Enfin, en 1984, un important amendement à l'Employment Act développe une conception particulièrement radicale de la flexibilité: il n'y a pas d'heures supplémentaires si le temps de travail n'excède pas 88 heures sur deux semaines, si chaque semaine ne dépasse pas 48 heures, ni chaque journée 12 heures; le repos hebdomadaire (un seul jour obligatoire) peut varier à volonté, si bien que le salarié peut avoir à travailler jusqu'à douze jours consécutifs ${ }^{105}$.

$\mathrm{Au}$ total on a donc un espace de négociation syndicats-patronat sévèrement limité (essentiellement les salaires et les primes, les congés, l'encouragement à la sécurité et à la productivité), et un espace de conflictualité encore plus restreint: dès 1955, 14 jours de préavis dans les secteurs "essentiels"; dès 1960, interdiction (Trade Disputes Act) des grèves de solidarité, des grèves contre le gouvernement, des grèves gênantes pour la population (en particulier des grèves générales, comme en 1963...), des piquets de grève "intimidants", et simultanément protection des non-grévistes, y compris syndiqués; l'arbitrage obligatoire du ministre, établi la même année par l'Industrial Relations Ordinance, peut intervenir dans tout conflit, à n'importe quel moment, sans avoir à être justifié, et rend toute grève illégale; en 1967, la grève est interdite dans les services publics. Par contre la latitude d'intervention du ministre du Travail et de l'IAC est extrême: les jugements de cette dernière (devant laquelle les plaignants ne sont en principe pas représentables par des avocats ${ }^{106}$, et dont le président peut à l'occasion siéger seul) ne sont même pas conditionnés par sa propre jurisprudence, et sont, sauf exception rarissime d'intervention de la Haute Cour, définitifs!107 L'IAC (partiellement relayée depuis 1981 par la Labour Relations division du ministère) joue un rôle essentiel dans la prévention des conflits: elle est à peu près systématiquement consultée à la moindre difficulté, avant comme pendant la négociation des

104Ibid., pp. 111-115

${ }^{105}$ Ibid., pp. 131-134

${ }^{106}$ C'est Lee Kuan Yew, lui-même récemment encore avocat, qui le propose en 1960 au Parlement: "une intervention venant d'un avocat, parfois habile, parfois moins habile, contribue seulement à retarder et à compromettre la conclusion appropriée et rapide d'une audience d'arbitrage. J'ai peur de devoir admettre qu'en principe nous devrions gagner du temps en excluant avocats et avoués" - Legislative Assembly Debates, State of Singapore, Official Report, vol. 12, 12 février 1960, p. 310

107Ibid., pp. 145-146 
conventions collectives; entre 1960 et 1983, elle est intervenue environ 2500 fois, dans plus de $95 \%$ des cas sur la demande des parties. Signe de la faible conflictualité entre partenaires sociaux "compréhensifs": entre 1967 et 1983, on n'a compté que 24 arbitrages obligatoires.

C'est que le syndicalisme indépendant du pouvoir a été contraint de se soumettre, et plus souvent de se démettre. Le PAP a été impitoyable, très ouvertement, avec ceux qui se mettaient en travers de son chemin, dans une collusion avérée avec le patronat. Ainsi, le 18 juillet 1963, on annonce officiellement que "à ce jour un total de 1149 travailleurs appartenant à la SATU ont perdu leurs emplois du fait de l'aventurisme politique des dirigeants pro-communistes"108. Quand, en octobre 1966, éclate une grève dans les services publics, dirigée par un syndicat affilié au NTUC, le Premier ministre demande une suspension de deux ans des revendications. Devant le refus des salariés, il engage 1300 jaunes, impose un arbitrage obligatoire, présente le projet d'amendement interdisant la grève dans les services publics, et menace d'expulser les 7000 non citoyens membres du syndicat, pour la plupart d'origine indienne; Lee va jusqu'à lancer devant le Parlement: "ils n'ont qu'à retourner dans leur pays"109. En janvier 1969, Lee, apprenant une grève du zèle des dockers, assure publiquement qu'il considère cela comme "une haute trahison"110, décide de licencier les dirigeants de la grève, et de les expulser de leurs logements de fonction ${ }^{111}$. En 1980, une grève du zèle des pilotes de Singapore Airlines est férocement brisée: leur syndicat, indépendant du NTUC, est désenregistré, ses dirigeants poursuivis ${ }^{112}$.

Les syndicats "officiels" ne jouent absolument pas le rôle d'un contre-pouvoir. Ils contribuent plutôt à l'échec des rares mouvements ouvriers authentiques. Ainsi lors de la grève de Metal Box, en 1977, dirigée par un petit syndicat indépendant. La direction du NTUC prétend que la grève est "condamnée à l'échec", denonce une "interférence étrangère" dans le soutien apporté aux grévistes par le syndicat des métallos australiens, et assure que "l'opinion publique sera contre une telle action": les grévistes osent en effet réclamer le licenciement $\mathrm{du}$ directeur du personnel, et protester contre le licenciement sans motif clair de 22 ouvriers! Un recours ayant été déposé devant le ministre du Travail, la grève en est doublement illégale: Devan Naïr lui-même appelle les ouvriers à démettre leurs représentants syndicaux (et, presque ouvertement, le patron à les licencier) et à se conformer aux procédures définies par l'IRA. Même quand le NTUC ne peut éviter d'intervenir, cela tient du "retenez-moi ou je fais un malheur", ou plus sérieusement de la

${ }^{108}$ Cité in Anantaraman, op. cité, p. 73

${ }^{109}$ Cité in Chan Heng Chee, Singapore: The Politics of Sorvival 1965-67, Oxford

University Press, 1971, p. 28

${ }^{110}$ Cité in Josey, op. cité, p. 493

${ }^{111}$ Margolin (1989), op. cité, p. 165

${ }^{112}$ Chris Leggett, "Singapore's Industrial Relations in the 1990s", in Garry Rodan, Singapore changes guard: social, political and economic directions in the 1990s, Melbourne, Longman Cheshire, 1993, p. 119 
manoeuvre dilatoire. Ainsi, en 1978, après l'explosion du pétrolier Spyros, en cours de nettoyage, qui fit 60 morts et de nombreux blessés, Devan Naïr ne peut faire moins qu'exprimer "son sentiment de stupéfaction et d'horreur... sa colère froide et sans pardon possible"113. On réclame "la punition des coupables", on rappelle les avertissements syndicaux répétés contre la très grande dangerosité de ce travail. On propose de mettre sur pied des comités de sécurité dans les entreprises les plus exposées aux accidents et, si rien n'avançait, horresco referens, d'arrêter le travail... Mais le NTUC se fie et participe à la commission gouvernementale d'enquête; présidée par un chef d'entreprise étranger, elle concluera à la responsabilité du Port de Singapour: le rapport sera en conséquence écarté, son premier signataire contraint à quitter le pays, et un peu plus tard un (authentique) lampiste sera condamné pour "negligence". Le temps a passé, le NTUC n'agira pas ${ }^{114}$.

Résultat: Singapour apparaît, par bien des côtés, un paradis de l'entreprise triomphante, soigneusement débarrassée par la contrainte étatique de tout risque de se voir opposé un quelconque contre-pouvoir; même l'église catholique -ou plutôt certains de ses courants- qui, après les étudiants du milieu des années soixante-dix, avait fait mine de se préoccuper de la défense des travailleurs, en particulier des immigrés, se voit sévèrement rappelée à l'ordre par une vague d'arrestations, en 1987115. Le principe de la toute-puissance patronale est acquis depuis les lois de 1968. La main d'oeuvre doit s'asservir par sa flexibilité maximale au rythme des machines et aux fluctuations des commandes. Quand les choses vont bien, il y a toujours loisir, malgré les réticences gouvernementales, de faire appel à quelques dizaines de milliers d'immigrés (ce ne sont pas les réserves qui manquent...) trop heureux de gagner à peu près leur vie. Quand tout va mal, ils seront licenciés les premiers, et ipso facto expulsés, sans que quiconque ne proteste: ainsi, lors de la récession de 1973-74, l'industrie électronique se débarrasse du quart de ses effectifs, soit une quinzaine de milliers de salariés ${ }^{116 ;}$ la crise, plus profonde, de 1985 entraîne 96000 suppressions d'emplois ${ }^{117}$ (dont 45000 dans le bâtiment, et l'essentiel du reste dans l'industrie), qui frappent pour les deux tiers des immigrés; le taux de chômage grimpe quand même de $2,7 \%$ à $4,9 \%$, ce qui représente un bond en avant d'une trentaine de milliers de personnes; de nombreuses entreprises ferment, ou licencient drastiquement ${ }^{118}$. Mais tout cela passe, apparemment, comme une lettre à la poste: aucune grève, aucune protestation voyante. La seule "action" d'abord entreprise par le NTUC, c'est, sur l'autel de la préservation de l'emploi, la renonciation, un syndicat après l'autre, aux augmentations de salaires initialement

${ }^{113}$ Labour News (organe du NTUC), 15 octobre 1978

${ }^{114}$ Margolin (1989), op. cité, pp. 190-191

${ }^{115}$ Ibid., pp. 262-263

116Ibid., p. 220

${ }^{117}$ Soit $7,7 \%$ des actifs, ce qui est énorme sur une durée aussi courte

${ }_{118}$ Ministry of Trade and Industry, Economic Survey of Singapore 1985, Singapour, 1986 , p. IX et $43-45$ 
prévues par le NWC...119 Il n'est guère payé de retour par le patronat: ainsi la compagnie Hydril Private Ltd licencie-t-elle neuf salariés, dont les trois principaux responsables locaux du Shipbuilding and Marine Employees' Union, syndicat membre du NTUC; ce dernier approuve le débrayage qui s'ensuit, deux jours durant, en janvier 1986: c'est la première grève depuis $1977^{120}$ ! Il est quand même significatif qu'elle soit motivé par la défense de cadres syndicaux, alors que, selon les données officielles, 511 entreprises ont licencié en 1985... Les salaires euxmêmes sont délibérément utilisés par l'Etat comme un élément central de la régulation macro-économique: au stop des années 1966-78 succède le go de $1979-82^{121}$, sans que les travailleurs y soient pour quoi que ce soit.

\section{Conclusion: renouveau de la résistance ouvrière ou déclin définitif?}

La façade du "système Singapour", et tout particulièrement de ses relations du travail, est lisse, sans aspérités, et ce depuis le milieu des années soixante. Tout semble parfaitement fonctionnel, huilé, tranquille. On peut néanmoins observer un certain nombre de phénomènes discrets, qui prouvent que des ouvriers peu disposés (quelles qu'en soient les raisons) à l'action collective n'en manifestent pas moins une remarquable capacité de résistance individuelle, au moins passive, face à la volonté avérée du pouvoir de les réifier en inputs purs et simples du développement économique ${ }^{122}$. Ce mouvement vibrionnant des salariés d'un emploi à l'autre, qu'on appelle à Singapour le job-hopping, en est le meilleur exemple: il montre la volonté de tirer parti au maximum du plein emploi quasi-constant pour négocier de complexes trajectoires ascendantes individuelles, en manifestant le plus grand mépris pour ces "valeurs" à la japonaise, ressassées par le pouvoir, que sont la fidélité à l'entreprise et la fierté du travail bien fait, à quelque niveau que ce soit ${ }^{123}$. La somme de ces phénomènes micro-économiques est macro-économiquement considérable: une pression à la hausse de la masse salariale, que le pouvoir peine de plus en plus clairement à maîtriser, depuis une décennie au moins. Tout se passe comme si l'ouverture des vannes salariales, en 1979, avait définitivement compromis l'idée même d'austérité124: quand le NWC, en 1983, cesse de recommander des

\footnotetext{
${ }^{119}$ Anantaraman, op. cité, p. 180

${ }^{120}$ Ibid., p. 135

${ }^{121}$ En moyenne $10 \%$ d'augmentation annuelle des salaires réels, de façon à remplacer

les industries de main d'oeuvre par des activités milieu ou haut de gamme

${ }^{122}$ "Je veux être sûr que tous les boutons fonctionnent", déclare Lee aux fonctionnaires peu après l'indépendance - Bakti, vol. III, n³ 3 , décembre 1965, p. 7

123"Un poseur de briques japonais est un poseur de briques fier et expérimenté. Un poseur de briques singapourien ne l'est pas" - Lee Kuan Yew, Press Release, 6 novembre 1979

${ }^{124}$ Cette seule explication serait évidemment trop simple; outre l'intégration du facteur plein emploi dans les anticipations de salariés dont la plupart désormais n'ont
} 
hausses importantes, le mouvement continue cependant, à peine ralenti. Il faudra le choc de 1985, et le ressurgissement momentané du chômage, jusqu'en 1988 (il est encore alors de 3,3\%), pour faire repasser l'augmentation des rémunérations en dessous de celle de la productivité; depuis 1989, cela n'a plus été le cas qu'en 1993 et, hormis cette année de récession internationale, la hausse des salaires réels n'a jamais été inférieure à 4\% l'an (5,2\% en 1994) ${ }^{125}$.

Rien n'y fait, et le pouvoir semble, sans trop l'avouer, se résigner à ne plus contrôler (ce qui ne veut pas dire qu'il n'ait plus les moyens de l'influencer) le mouvement des salaires. Ce n'est pas faute d'avoir essayé. Lee, depuis 1977, exhorte inlassablement ses compatriotes à suivre le "modèle japonais", repeint plus récemment en "valeurs asiatiques", à conserver ou restaurer leur inclination ancestrale supposée à la frugalité, à l'abnégation, au dévouement à la collectivité; mais, après une dizaine d'années d'efforts, il a fallu en 1989 se résoudre à supprimer des programmes scolaires l'enseignement religieux (le confucianisme pour les jeunes Chinois non chrétiens), décrié et peu efficace. A côté de ces stimulants moraux, le NWC a cherché à mettre en place des stimulants matériels. Depuis 1977 (année marquant aussi le début du Look East en direction du Japon), il vise, on l'a vu, à sanctionner les travailleurs déméritants; mais les augmentations qui leur seraient déniées seraient à répartir entre leurs collègues les plus valeureux - rien de cela ne fut jamais appliqué. En 1980, il propose, plus modestement, de mettre à part 3\% de la masse salariale, à répartir entre ces derniers - l'idée est abandonnée en 1982. Toujours en 1980, il suggère de supprimer la part patronale de la contribution au Central Provident Fund (caisse de retraite) pour les salariés embauchés depuis moins d'un an - le gouvernement n'ose pas le suivre ${ }^{126}$. Le président du NWC, l'économiste Lim Chong Yah, tente de rationaliser ces échecs persistants: "Le principe de la récompense des meilleurs travailleurs n'est pas en question, mais son implémentation s'est avérée pratiquement impossible. Cela a généré un grand malaise (considerable unhappiness) parmi les travailleurs, dans la mesure où le résultat est souvent perçu comme inéquitable. Pour les employeurs, l'identification des "travailleurs supérieurs à la moyenne" a été une tâche impossible (an exercise in futility), étant donné le caractère inadéquat du système le plus courant d'appréciation de la performance"127. De notre point de vue, l'intérêt est la mention du mécontentement efficace des salariés; un autre signe de ce dernier est l'extension fréquemment réalisée par les entreprises du groupe des "meilleurs" de $20 \%$ environ de la main d'oeuvre (ce qui était prévu par le

pratiquement rien connu d'autre, il faudrait incriminer la complexification et la diversification d'une économie rapidement modernisée, ainsi que le poids sans cesse croissant dans la main d'oeuvre des qualifications supérieures, particulièrement aptes à valoriser au maximum leur avantage comparatif

125Ministry of Trade and Industry, Economic Survey of Singapore 1994, Singapour, 1995 , pp. $17-18$

${ }^{126}$ Anantaraman, op. cité, pp. 126-127

${ }^{127}$ Straits Times, 14 avril 1982, cité in Anantaraman, op. cité, p. 177 
NWC) à $70 \%$, voire plus - ce qui par ailleurs ne laisse plus qu'un avantage salarial minime aux bénéficiaires ${ }^{128}$.

L’impasse entraîne, de la part du gouvernement, une décision radicale: l'annonce, dès 1982, de son retrait progressif du NWC, ce qui devrait inciter à une décentralisation des négociations salariales, s'agissant en particulier des grandes entreprises. L'habitude est cependant si ancrée d'attendre les sacro-saintes recommandations annuelles qu'il faut se résoudre, en 1986, à supprimer carrément celles-ci, et à réduire le NWC au rôle d'un organisme d'études et de conseil aux employeurs et aux syndicats, avec néanmoins une phase transitoire de cinq ans. Le NWC nouvelle manière tout comme le Report of the Economic Committee (1986) dirigé par le nouveau ministre du Commerce et de l'Industrie et fils de Lee Kuan Yew, Lee Hsien Loong, insistent à nouveau sur l'importance d'une flexibilité maximale des rémunérations ${ }^{129}$, mais que chaque entreprise se chargerait d'organiser par elle-même; quelques mesures précises sont cependant préconisées: l'accord d'une prime de $2 \%$ aux salariés les plus stables; l'abandon de l'avancement à l'ancienneté; l'ouverture de 1 à 1,5 de l'échelle salariale pour chaque type de poste. Mais seul le secteur public essaiera sérieusement de se relancer dans la quasi-quadrature du cercle qu'est l'évaluation du "mérite". Il est significatif que ce qui surnagera de la quête de flexibilité sera surtout l'idée -très japonaise elle aussi- d'une part variable de la masse salariale, autour de $20 \%$ en moyenne, distribuée sous forme de primes en fonction des résultats annuels (productivité et/ou profit), mais à tous les salariés, sans considération de leur performance individuelle ${ }^{130}$.

Depuis 1981 (recommandation du National Productivity Board) et surtout 1982 (résolution du 1er Mai du NTUC), la tendance à l'éclatement de la gestion de la force de travail au niveau de la firme est corroborée par la nouvelle stratégie visant à créer un maximum de syndicats d'entreprise (house unions) aux dépends des vastes regroupements industriels, parfois sans limites claires, et constituant de véritables Etats dans l'Etat'131 (cf ci-dessus). Dès octobre 1982 les premiers syndicats d'entreprise sont constitués, les fédérations "attrape-tout" PIEU et SILO sont dissoutes. La pression de la direction du NTUC sur les syndicats récalcitrants (en particulier ceux du pétrole -cf ci-dessus- et des transports aériens) est très forte (y compris par des actions disciplinaires), en particulier en 1984, et elle a gain de cause ${ }^{132}$. Des syndicats décentralisés, mais créés par en haut, contre l'avis des militants concernés, et toujours fortement reliés à la direction confédérale ! On est assez loin en fait du corporatisme d'entreprise à la japonaise, fondé sur un authentique compromis local entre salariés et patronat. On en est encore plus loin -mais l'on est plus près du

128Ibid., note 29, pp. 192-193

${ }^{129} 1990$ Singapore Yearbook of Labour Statistics, op. cité, p. 22

${ }^{130}$ Anantaraman, chapitre 11, pp. 173-200

${ }^{131}$ Ils représentaient un danger pour la "vieille garde" du NTUC, comme venait de le démontrer l'affaire de l'ambitieux et corrompu Phey Yew Kok

${ }^{132}$ Anantaraman, op. cité, pp. 207-208 et note 32, pp. 212-213 
corporatisme "organique" inventé par le fascisme- si l'on ajoute que, dès 1984, les cadres dirigeants peuvent devenir membres des syndicats d'entreprise, et qu'en 1991 on décide de les admettre dans les autres syndicats. Ce à quoi s'oppose la Singapore National Employers' Federation, qui défend paradoxalement, à cette occasion l'idée d'intérêts (on pourrait ajouter "de classe") irréductiblement contradictoires ${ }^{133}$.

Et cependant, là encore, sans qu'aucune opposition soit clairement discernable, le bilan, au début des années 1990, n'est pas très convaincant: en 1980, 60,2\% des syndiqués appartenaient à des organisations comprenant plus de 10000 adhérents; c'est encore le cas de $41,2 \%$ en 1990. Quant aux syndicats correspondant à la taille de la plupart des entreprises (moins de 1000 adhérents), ils comprenaient $8,5 \%$ des syndiqués en $1980,7 \%$ en 1990 . Et le nombre total des unions est exactement le même (83) aux deux dates ${ }^{134}$. On a, semble-til, plutôt un échec de la décentralisation syndicale, qui s'explique sans doute par la faiblesse des "cultures d'entreprise" à Singapour, et par la très grande centralisation conservée par son système de régulation économico-politique, largement aux mains d'un petit groupe de dirigeants, au vu et au su de tous. Il faudrait, dans le même sens, citer le Company Welfare Scheme (ou Cowec), lancé en 1984 à grand renfort de publicité: il visait à faire gérer directement par l'entreprise, au bénéfice de ses salariés, $10 \%$ de la contribution patronale au CPF. Aujourd'hui le Cowec a presque partout été abandonné...

On ajoutera enfin que les travailleurs singapouriens "votent avec leurs pieds" leur défiance à l'encontre du NTUC (sans, bien sûr, que ce qu'ils voudraient mettre à la place soit clair, et sans doute guère plus pour eux que pour nous). On est loin des 35,9\% d'actifs syndiqués (à la SATU surtout) de la fin 1962; les syndiqués (désormais presque tous au NTUC) sont $18,3 \%$ des actifs en $1969,21,9 \%$ en 1980 , mais $14,3 \%$ en 1990 (212 000, contre 244000 dix ans plus tôt). Ce sont dans les finances et les services (39\% de syndiqués) et dans les transports $(32,1 \%)$ qu'ils sont les plus nombreux: on tombe à $15,2 \%$ dans l'industrie, à $5,2 \%$ dans le commerce... et à $0,5 \%$ dans le bâtiment, place forte de l'immigration ${ }^{135}$. On peut rapprocher ces données d'une étude, malheureusement ancienne (1970), sur le NTUC: parmi ses secrétaires de syndicats, on compte $51 \%$ d'Indiens ( $8 \%$ de la population) pour $37 \%$ de Chinois $(75 \%)$; seuls $37 \%$ des syndicats regroupent plus de $40 \%$ d'adhérents de moins de trente ans, alors que ceux-ci constituent alors $46 \%$ de la main d'oeuvre ${ }^{136}$. on discerne un syndicalisme regroupant typiquement des cols blancs, relativement âgés, très souvent employés des services publics ou des transports (les bastions des Indiens), où la tradition corporatiste, anglophone et politiquement modérée domine. Par contre les gros bataillons ouvriers

${ }^{133}$ Leggett, art. cité, p. 123

${ }^{134}$ Yearbook, op. cité, pp. 51-52

${ }^{135}$ Ibid., p. 53

136Noeleen Heyzen, Trade Union Leaders in Singapore, Singapour, 1970 
sont largement absents: en 1990, l'industrie et le bâtiment ne fournissent que 30\% des adhérents du NTUC.

Et cependant... Un mal mortel menace cette classe ouvrière "en pointillés": sa disparition progressive, tout simplement. Le phénomène n'est certes pas aussi voyant, aussi rapide que le fut, des années soixante à 1980, l'inflation numérique des travailleurs de l'industrie (cf ci-dessus): l'expansion économique est telle qu'il n'y a pas diminution en chiffres absolus des ouvriers d'usine (255000 en 1980, 256000 en 1994), et que les actifs du secteur secondaire se sont renforcés: 313 000 dans l'industrie en 1980, 422000 en 1994; si l'on adjoint les actifs du bâtiment, la progression apparente est plus vive encore: ils passent de 58000 en 1980 (cela donne au total 370 000) à 108000 en 1994 (530 000). Mais, depuis maintenant près de quinze ans, la part du secondaire dans la population active a assez nettement diminué (de $34,6 \%$ à 32,2\%; de 29,2\% à 25,6\% pour la seule industrie, alors que le bâtiment -à vrai dire très tiré en avant par le boom des services- est passé de $5,4 \%$ à $6,6 \%$, malgré son quasi-effondrement du milieu des années quatre-vingt). Le plus frappant est cependant la considérable redistribution des cartes au sein même de la main d'oeuvre industrielle: les ouvriers en représentaient $81,7 \%$ en 1980 , mais seulement $60,7 \%$ en 1994. Par contre les détenteurs de fonctions administratives y ont grimpé de $3,8 \%$ à $8,8 \%$; les professionnels, ingénieurs et techniciens y ont bondi de 4,5\% à 18,5\%: les employés de bureau, plus modestement, sont passés de $7 \%$ à $11,1 \%$ des effectifs. Dans le bâtiment lui-même, la proportion d'ouvriers a baissé, quoique plus modestement, de $76,2 \%$ à $65,8 \%$ (les gains de productivité sont loin d'y égaler ceux de l'industrie).

L'effet d'ensemble est impressionnant: de 1980 à 1994, les ouvriers de l'industrie sont passés de $23,9 \%$ à $15,5 \%$ des actifs; si on leur adjoint leurs confrères du bâtiment (passés, eux, de 4,1\% à 4,3\% des actifs), cela donne $28 \%$ de la main d'oeuvre en $1980,19,8 \%$ en 1994 - soit moins que les $21,5 \%$ de 1957 (cf introduction), avant le début de la croissance industrielle rapide ${ }^{137}$. Même en admettant qu'un certain nombre de travailleurs immigrés ne soient pas déclarés (dans le bâtiment surtout), l'évolution d'ensemble n'en reste pas moins parfaitement claire. Premièrement, les ouvriers, divisés, baillonés, ne sont même plus clairement hégémoniques numériquement dans leurs usines: on n'est pas loin d'y compter un cadre ou technicien pour deux travailleurs de base, contre un pour dix il y a seulement quinze ans. Leur capacité à agir ensemble, si virtuelle soit-elle dans le contexte singapourien, est encore amenuisée par leur déconcentration relative: les PMl ont davantage cru que la grande industrie, et en conséquence la proportion des ouvriers figurant dans des entreprises inférieures à dix salariés est passée de 7,7\% en 1980 à 13,7\% en 1994.

${ }^{137}$ Economic Survey, op. cité, 1980 et 1994 
Deuxièmement: en 1957, les ouvriers, peu nombreux, parvenant mal à se distinguer de la vaste masse prolétarienne, pouvaient cependant attendre à certaines occasions le soutien de couches très proches d'eux par la pauvreté, la formation (ou plutôt la faiblesse de celle-ci) et le mode de vie; en 1994, une grande partie des "cols blancs" s'en distinguent au contraire, car ils sont mieux rémunérés, mieux formés, et plus à même de profiter de l'offre consumériste, voire culturelle, qui s'est énormément diversifiée. Le fossé entre citoyens et non-citoyens s'est également considérablement élargi. Les ouvriers d'aujourd'hui, aussi faibles numériquement que dans les années cinquante, se trouvent ainsi objectivement beaucoup plus isolés. Rien de tout ceci n'est inconnu, en notre ouest du monde. L'extraordinaire est plutôt que, à partir d'une situation si différente il y a encore si peu de temps, Singapour soit déjà si "banalement" proche des pays dits avancés d'occident. Le grand port sous-développé d'avant-hier, le "nouveau pays industriel" d'hier apparaît désormais plutôt "nouveau pays post-industriel", au prix d'une prodigieuse accélération de nos normes d'évolution. Entre ses balbutiements encore proches et son actuel déclin, la classe ouvrière singapourienne n'aura-t-elle connu qu'une étroite fenêtre d'opportunité, sans être capable d'en tirer parti?

Jean-Louis Margolin Université de Provence (Aix) 